\title{
Terpenes/Terpenoids in Cannabis: Are They Important?
}

\author{
Lumír Ondřej Hanuš Yotam Hod \\ Lumir Lab, Asana Bio Group Ltd., The Hadassah Medical Center, Hebrew University Biotechnology Park, \\ Ein Kerem, Jerusalem, Israel
}

\section{Keywords}

Cannabis · Cannabis sativa L. · Gas chromatography-mass spectrometry $\cdot$ Terpenes $\cdot$ Terpenoids

\begin{abstract}
Cannabis sativa plant has not only cannabinoids as crucial compounds but also the other compounds that play important role as synergistic and/or entourage compound. Cannabis/hemp plant materials and essential oils were analyzed with the help of gas chromatography/mass spectrometry detector for the content of terpenes and terpenoids. The main terpenes/terpenoids and their abundance in the samples were evaluated. Results of this study will be helpful in the next evaluation of these compound in mixture with cannabinoids and their importance in medical treatment.
\end{abstract}

(c) 2020 The Author(s)

Published by S. Karger AG, Basel

\section{Introduction}

Since the past 25 years, the interest in cannabis (Cannabis sativa L.) has been growing extremely worldwide. The main reason behind such growing interest is not the treatment of some serious illnesses with cannabis but rather the desire to get rich quickly.

karger@karger.com www.karger.com/mca

Karger"

\section{(C) 2020 The Author(s)}

Published by S. Karger AG, Basel

This article is licensed under the Creative Commo NonCommercial-NoDerivatives 4.0 International License (CC BYNC-ND) (http://www.karger.com/Services/OpenAccessLicense). Usage and distribution for commercial purposes as well as any distribution of modified material requires written permission.
Although medical cannabis in some countries is considered legal, it is not yet a pharmaceutical drug. There are 3 main reasons. (a) Fear and stigma - 80 years of prohibition accompanied with negative propaganda. (b) Lack of standardization - since it is not a single-molecule drug. Cannabis in fact is a plant of over 1,000 chemical constituents, varying by chemotype (chemical phenotype) batch and crop. Sometimes, people incorrectly referred to chemotype as strain, variety, cultivar, or chemovar. Chemotypes are plants of the same genus that are virtually identical in appearance but produce essential oil with different major constituents. Chemotypes are variants within a single botanical species. Today, there are thousands of "strains," many of which have similar names but cultivated in different climatic regions, and each with unique chemical ingredient profile that activates differently. (c) The cart before the horse - cannabis became legal and approved without standard clinical trials. Now, we have to "back in" to the efficacy to see what type of cannabis works for which medical conditions [1].

Cannabis use as medicine goes back for thousands of years [2], but after the USA started to fight against it since 1937, it disappeared from pharmacopeias all over the world. Fortunately, in 1950, Krejčí [3, 4] from Czechoslovakia discovered antibiotic principle of cannabis and they started to use cannabis clinically at hospitals. Back in 1954, the first scientific conference under "Cannabis as a

Lumír Ondřej Hanuš

Lumir Lab, Asana Bio Group Ltd., The Hadassah Medical Center Hebrew University Biotechnology Park, Ein Kerem Campus Jerusalem 91120 (Israel) lumir@lumirlab.com 

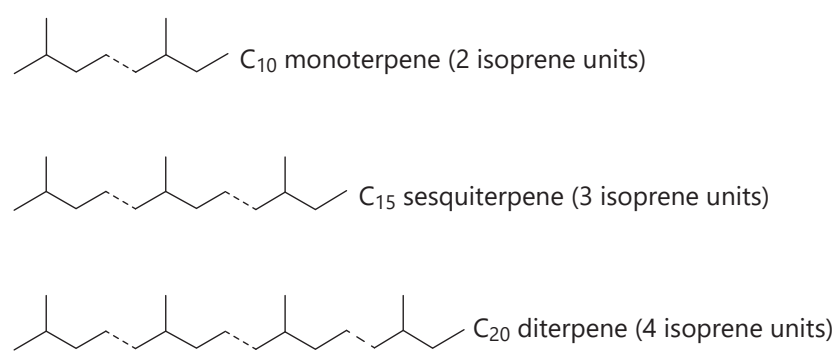

Fig. 1. Linking isoprene units "head to tail" to form terpenes/terpenoids.

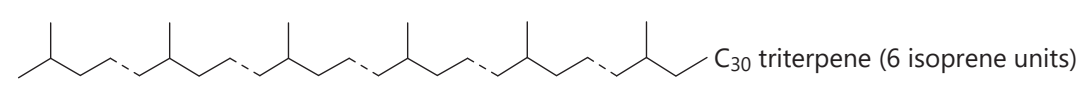

medicine" was held in Olomouc, Czechoslovakia [5]. Krejčí and Šantavý [6] identified this antibiotic principle compound of cannabis, which is effective against grampositive microorganisms and some pathogens. They named the compound responsible for this effect cannabidiolic acid [6-8]. It was the first real cannabinoid isolated from $C$. sativa $\mathrm{L}$. From that time, cannabis grown in Czechoslovakia (mostly the Czechoslovak chemotype Rastislavice) was used for the treatment in the Faculty hospital of Olomouc $[9,10]$ up to 1990.

The first cannabinoid compound isolated and identified from hemp was cannabinol [11-13]. This compound in fact is an artifact, which originates from $\Delta^{9}$ tetrahydrocannabinol (THC) in the plant resin and after plant harvest, storage, or heating [14]. Decarboxylation product of cannabidiolic acid, cannabidiol (CBD), was fully identified in 1963 [15, 16, 19]. Psychotomimetically active compound of cannabis, THC, was fully characterized in 1964 [17-19]. Today, 177 cannabinoid compounds are known in C. sativa L. [20-23]. Many of them are certainly artifacts originating during harvest, drying, and workup of cannabis plant. After the identification of the above-mentioned cannabinoids, research started on these compounds. Cannabinoid receptors, CB1 [24] and CB2 [25], were discovered in the human body. Full understanding of the whole endocannabinoid system in the human body gave an isolation of natural ligands (today called endocannabinoids) [26-31]. This brought us to understand the medicinal value of cannabis plant, which was used for millennia in treatment [2], and today, it is being legalized for the treatment of different diseases in many countries. Because of illegalization and stigmatization of this plant, there is still a lot of work to be accomplished to acquire full knowledge of the medicinal power of this plant [32].
The first scientific study conducted on cannabis contents goes back to the first half of the 19th century [33]. Bolas and Francis [34] made the first attempt to identify the compounds in the commercial resinous extract of Indian hemp in 1869. They isolated oxy-cannabin, $\mathrm{C}_{5} \mathrm{H}_{6} \mathrm{O}_{2}$, and acid compound, which crystallize in plates.

It was almost generally accepted that the only active compounds in cannabis are compounds typical for this plant, cannabinoids. Only in the last several years scientists started to speculate about synergic and/or entourage effect of the other cannabis compounds. In the first row today are terpenes/terpenoids, but our plans are also to study flavonoids, flavonoid glycosides, and also polyphenols. It is very likely that the first isolated terpene was just $\beta$-caryophyllene.

As workers in Italian hemp fields became gay and giddy from a volatile principle of hemp, Valente searched for the compound that causes it. He employed steam distillation of the fresh leaves of hemp cultivated for fibers to obtain sesquiterpene from essential oil, with the empirical formula $\mathrm{C}_{15} \mathrm{H}_{24}$ [35-37]. It was probably $\beta$ caryophyllene which is the most common main sesquiterpene in hemp. Vignolo [38, 39] also used distillation in a current of steam to prepare essential oil and subsequently the same sesquiterpene $\mathrm{C}_{15} \mathrm{H}_{24}$ as Valente. Wood [40] isolated from charas (because it contains no chlorophyll) a monoterpene $\mathrm{C}_{10} \mathrm{H}_{16}$ (probably myrcene that is usually the main monoterpene in hemp) and a sesquiterpene $\mathrm{C}_{15} \mathrm{H}_{24}$ ( $\beta$-caryophyllene?). Simonsen and Todd [41] were the first to name isolated terpene. They extracted $p$-cymene $\left(\mathrm{C}_{10} \mathrm{H}_{16}\right)$, p-cymenene $\left(\mathrm{C}_{10} \mathrm{H}_{12}\right)$, and humulene $\left(\mathrm{C}_{15} \mathrm{H}_{24}\right)$ from Egyptian hashish.

If the question is what are terpenes or terpenoids in cannabis plant, the answer is terpenes are hydrocarbons and terpenoids are oxygen-containing terpenes. They can 
Table 1.

\begin{tabular}{ll}
\hline Monoterpenes $-\mathrm{C}_{10} \mathrm{H}_{16}$, mw 136 & Monoterpenoids \\
\hline Acyclic $(\beta$-myrcene) & Acyclic (linalool $-\mathrm{C}_{10} \mathrm{H}_{18} \mathrm{O}$, mw 154) \\
Monocyclic ( $\alpha$-phellandrene) & Monocyclic (cis-linalool oxide $-\mathrm{C}_{10} \mathrm{H}_{18} \mathrm{O}_{2}$, mw 170) \\
Bicyclic ( $\alpha$-pinene) & Bicyclic (cis-sabinene hydrate $-\mathrm{C}_{10} \mathrm{H}_{18} \mathrm{O}$, mw 154) \\
Tricyclic (tricyclene) & \\
\hline
\end{tabular}

Table 2.

\begin{tabular}{ll}
\hline Sesquiterpenes $-\mathrm{C}_{15} \mathrm{H}_{24}$, mw 204 & Sesquiterpenoids \\
\hline Acyclic (cis- $\beta$-farnesene) & Acyclic (cis-nerolidol $-\mathrm{C}_{15} \mathrm{H}_{26} \mathrm{O}$, mw 222) \\
Monocyclic ( $\alpha$-humulene) & Monocyclic (humulene epoxide II - $\mathrm{C}_{15} \mathrm{H}_{24} \mathrm{O}$, mw 220) \\
Bicyclic $(\beta$-caryophyllene) & Bicyclic (sesquicineole $-\mathrm{C}_{15} \mathrm{H}_{26} \mathrm{O}$, mw 222) \\
Tricyclic ( $\alpha$-cubebene) & Tricyclic (epi-cubebol - $\mathrm{C}_{15} \mathrm{H}_{26} \mathrm{O}$, mw 222) \\
\hline
\end{tabular}

Table 3.

\begin{tabular}{ll}
\hline Diterpenes & Diterpenoids \\
\hline Monocyclic $\left(m\right.$-camphorene $-\mathrm{C}_{20} \mathrm{H}_{32}$, mw 272) & Acyclic (phytol $-\mathrm{C}_{20} \mathrm{H}_{40} \mathrm{O}$, mw 296) \\
\hline
\end{tabular}

Table 4.

\begin{tabular}{ll}
\hline Triterpenes & Triterpenoids \\
\hline Acyclic (squalene $\left.-\mathrm{C}_{30} \mathrm{H}_{50}, \mathrm{mw} 410\right)$ & Tetracyclic (sitostanol $-\mathrm{C}_{29} \mathrm{H}_{52} \mathrm{O}$, mw 416) \\
& Pentacyclic (friedelin $\left.-\mathrm{C}_{30} \mathrm{H}_{50} \mathrm{O}, \mathrm{mw} 426\right)$ \\
\hline
\end{tabular}

be visualized as the result of linking isoprene units "head to tail" to form chains, which can be arranged to form rings (Fig. 1). It is the so-called biogenetic isoprene rule or the $\mathrm{C}_{5}$ rule [42].

The name terpene was suggested by Kekulé [43] for $\mathrm{C}_{10} \mathrm{H}_{16}$ hydrocarbons in 1866 . In C. sativa $\mathrm{L}$., plant terpenes/terpenoids can be divided into several groups. Here are examples of different types of terpenes (110 published up-to-date) and terpenoids (121 published up-todate) in this plant - examples of terpenes/terpenoids from cannabis of this particular group are in given parentheses (Tables 1-4):

Cannabinoids are derived from diterpene structure. The monoterpenes present in cannabis plant have another functional moiety like alcohol (fenchyl alcohol, linalo- ol, and borneol), aldehyde (neral), ketone (carvone), ester (bornyl acetate and linalyl acetate), ether (1,8-cineol), and phenol (thymol and carvacrol). The sesquiterpene molecules include structures like alcohols (farnesol) or ketones (nootkatone). To understand the full effects of terpenes/ terpenoids, it is necessary to explain some terms.

\section{Synergy}

The term synergy comes from the Attic Greek word бuvepyia (synergía, "collaboration"), which is based on

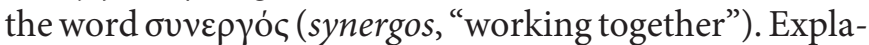
nation is easy. When we have 2 active compounds, they work together better than each one separately, which can be expressed by strict inequality: $1+1>2$. 


\section{Entourage Effect}

The term entourage effect was introduced in 1988 by Mechoulam and colleagues[44] and was explained as increased activity of an active compound with an inactive one, which can be expressed by strict inequality: $1+0>1$. Standardized cannabis drug preparations, rather than pure cannabinoids, could be generally considered the preferred ones $[45,46]$. We believe that all components of the cannabis plant likely exert some therapeutic effect, more than any single compound alone. There is increasing evidence that these compounds work better together than in isolation and that is exactly what is called today "entourage effect."

The analysis of the sample and the interpretation of the results of the analysis must be evaluated very responsibly. We do not have enough results in this field yet. Chemotype, location of cultivation, conditions of cultivation, season of cultivation, weather and microclimate, stage of plant development, method of processing after harvest, method of storage, storage time before analysis, part of a plant for analysis, and method of sample processing for analysis - all these parameters affect the results of the analysis. So far, changes in the content of cannabinoid substances during the growing season have been studied [47-50] and also dynamics of changes of cannabinoids and terpenes/terpenoids during vegetation period [51, 52].

Terpenes/terpenoids have a wide range of biological and pharmacological activities, for instance, antifungal, antiviral, anticancer, anti-inflammatory, antihyperglycemic, antiparasitic, antioxidant, and antimicrobial. It is not possible to describe all pharmacological effects of terpenes/terpenoids in this paper, but we shall give just some examples for imagination of how important these compounds are in this plant. Monoterpene myrcene is the smallest terpene but the most prevalent terpene found in most varieties of cannabis. Chemotypes high in myrcene will result in a "couch lock" effect (if a sample has over $0.5 \%$ myrcene), while chemotypes with low levels of myrcene $(<0.5 \%$ myrcene) will produce a more energetic high. It is simply the amount of myrcene that is in the sample that dictates how you will be affected. Myrcene is simply the important monoterpene in the plant. Myrcene has antipsychotic, antioxidant, analgesic, anti-inflammatory, sedative, muscle relaxant, and anticancerogenic properties [53-56]. The most important sesquiterpene in the cannabis plant is probably $\beta$-caryophyllene. It is a spicy terpene. This compound is the only terpene known to interact with the body's endocannabinoid system (selectively binds to the $\mathrm{CB}_{2}$ receptor) [57]. Caryophyllene has gastroprotective, analgesic, anticancerogenic, antifungal, antibacterial, antidepressant, anti-inflammatory, antiproliferative, antioxidant, anxiolytic, analgesic, and neuroprotective effects $[58,59]$. The presence of $\beta$-caryophyllene in many essential oils might contribute strongly to their antiviral ability. $\beta$-Caryophyllene displayed a high selectivity index of 140 against herpes simplex virus type 1 in vitro. The selectivity index was determined by the ratio of the cytotoxic concentration of the drug that reduced viable cell number by $50 \%$ to antiviral activity, which inhibited plaque numbers by $50 \%$ compared with the untreated control [60]. $\alpha$-Pinene (antibacterial, anti-inflammatory, bronchodilator, antiseptic, and gastroprotective) and $\beta$-pinene (antiseptic) are the next important compounds [61]. It is not possible to mention here all the biodynamic terpenes. After all, there may be mentioned, for instance, limonene (antibacterial, gastroprotective, antiproliferative, antifungal, anxiolytic, antidepressant, antimicrobial, antispasmodic, or immunostimulant) [62, 63]. Linalool (sedative, antipsychotic, anticonvulsant, anxiolytic, anesthetic, antidepressant, analgesic, antiepileptic, and antineoplastic) [64], terpineol (antioxidant, antibiotic, and relaxing effect) [65], or caryophyllene oxide (analgesic, anticancer, antifungal, and anti-inflammatory) [66]. Between others are also phellandrene (antifungal and digestive disorders) [67], ocimene (antifungal) [68], camphene (cardiovascular disease) [69], guaiol (antitumor) [70, 71], a-humulene (antibacterial, anti-inflammatory, and antitumor) [7274], $\gamma$-terpinene (analgesic, anti-inflammatory, antimicrobial, and anticancer) $[75,76], \beta$-elemene (antitumor, antineoplastic, and anticancer) [77-80], nerolidol (antiparasitic and antileishmanial) [81, 82], or citral (antifungal, antimicrobial, antiproliferative, cytotoxic, anticancer, and antitumor) [83-90].

Terpenes/terpenoids are largely responsible for the characteristic aroma of cannabis. An essential oil (mostly terpenes or terpenoids), especially when distilled, is not necessarily identical in its chemical composition with the oil that is present in the living plant. Quite often very high-boiling or low-boiling chemicals are simply "lost" due to the nature of the distillation process and due to economic and time constrains. Although most constituents remain intact during distillation, a few undergo chemical changes. Oil also contains substances that are formed from reactive precursors on distillation. The variation in essential oil composition may be due to factors that affect the plant's environment, such as geographical location, weather conditions, soil type, fertilizer used, the
Hanuš/Hod 
age of the plant, and the time and weather of day or year when it is harvested. Degradation tends to occur on prolonged storage, under poor storage conditions, or when the essential oil is otherwise exposed to air. Atmospheric oxygen can change the chemical composition of an essential oil by reacting with some of its constituents. Oxidation can also affect the efficacy of an essential oil. It can render an essential oil more hazardous [91]. When we use plant material, one must take attention to keep it protected from UV and air oxidation. Otherwise, some terpenes can transform to allergens $[92,93]$.

The first who pointed out the possible synergic and/ or entourage effect of cannabinoids and terpenes is Russo [94]. Only few studies have pharmacology, and further studies have to be accomplished prior to understanding the interactions of cannabinoids and terpenes/ terpenoids in humans. Well-arranged review on the terpene biosynthesis in cannabis plant was demonstrated by Kovalchuk et al. [95]. Terpene synthases from C. sativa were characterized [96-98]. Rice and Koziel [99] studied the odorous compounds (as terpenes/terpenoids as the other volatiles). Hazekamp et al. [100] gave a deeper understanding of cannabis effects in laboratory and clinical studies and the usefulness of a terpene/terpenoid approach for chemotaxonomic mapping of cannabis varieties for medicinal use. Gallily et al. [101] investigated the antioxidant and anti-inflammatory properties of 3 different terpene/terpenoid-rich hemp essential oils. They concluded that terpenes/terpenoids may be used to diminute acute inflammation effect, whereas the cannabinoids to inhibit chronic inflammation symptoms. Anti-inflammatory potential of terpenes present in cannabis was studied by Downer [102]. Current evidence of medicinal properties of terpenes was given by Baron [103] and Nuutinen [104] in their reviews. Entourage effect of terpenes/terpenoids and cannabinoids and their pharmacological activity were also studied by Namdar et al. [105] and Ferber et al. [106]. Blasco-Benito et al. [46] extracted fresh cannabis flowers in ethanol, after evaporation, followed by magnetic stirring on hot plate, which achieved cannabinoid decarboxylation. The extract (in $\mathrm{mg} / \mathrm{g}$ ) contains 3.4 THCA, 551.3 THC, 3.7 CBG, and no THCV, CBD, CBDA, cannabinol, and $\mathrm{CBC}$ and the 5 main terpenes were $1.9 \beta$-caryophyllene, 0.6 humulene, 0.4 nerolidol, 0.6 linalool, and $0.3 \beta$-pinene. While the ethanolic extract of cannabis flowers has higher antitumor activity than pure THC, this effect was not attributed to any of the 5 most abundant terpenes (THC and the 5 main terpenes in appropriate concentrations did not have higher activity than pure THC). Finlay et al. [107] tried to determine whether terpenes (myrcene, $\alpha$ - and $\beta$-pinene, $\beta$-caryophyllene, and limonene) in the cannabis plant have detectable receptor-mediated activity or modify the activity of THC, CBD, or the endocannabinoid 2-arachidonoylglycerol at the cannabinoid receptors. This study proves that the putative entourage effect cannot be explained by direct effects at CB1 or CB2. Nuutinen [104] has published a comprehensive paper on the medicinal properties of a number of cannabis mono- and sesquiterpene/terpenoids. These were studied in vitro, on animals, and in clinical trials. Performed studies have shown antimutagenic, antidiabetic, anti-inflammatory, analgesic, antioxidant, antibiotic, anticonvulsive, anticancer, antidepressant, anxiolytic, antitumor, neuroprotective, anti-allergic, and others.

\section{Experimental}

Plant Material and Essential Oils

All 54 chemotype inflorescence samples of cannabis were purchased from Israeli growers. Eight essential oils were of hemp harvested in August/September 2016 in the pre-Alpine region of Slovenia (Upper Savinja Valley), latitude NS $46^{\circ} 20^{\prime}$ 29.525 and longitude E $14^{\circ} 50^{\prime} 0.777$. These samples of essential oils were prepared by steam distillation of female flowers (upper third of the plant). One sample was from Czech Republic (Bialobrzeskie). Essential oils of cannabis were bought from Cali terpenes (45 samples).

\section{Sample Preparation}

A $20 \mathrm{mg}$ of plant material was extracted with hexane, filtered with Filter Fix ( $25 \mathrm{~mm}, 0.45 \mu \mathrm{m}$ Nylon Syringe Filter; SOLUFIX - SIMPLEPURE PTFE, $0.45 \mu \mathrm{m}$ ), and dissolved 20 times with hexane. Essential oil was diluted with hexane to a concentration of 0.1 $\mathrm{mg} / \mathrm{mL}$.

\section{Conditions of the Analysis}

Instrument: GC/MS (Agilent 7890B GC, Agilent 5977B MSD, PAL 3 [RSI 85]).

Column: Agilent Technologies, Inc., HP-5MS UI, $30 \mathrm{~m} \times 0.25$ $\mathrm{mm}$, film $0.25 \mu \mathrm{m}$.

Experimental conditions: At first, the column was held at $35^{\circ} \mathrm{C}$ for $5 \mathrm{~min}$, and afterward, the temperature was raised to $150^{\circ} \mathrm{C}$ at $5^{\circ} \mathrm{C} / \mathrm{min}$, then at $15^{\circ} \mathrm{C} / \mathrm{min}$ up to $250^{\circ} \mathrm{C}$, hold time $90 \mathrm{~min}$ (the inlet temperature was fixed at $250^{\circ} \mathrm{C}$; the detector at $280^{\circ} \mathrm{C}$; split injection $1: 5$; initial temperature $-100^{\circ} \mathrm{C}$; initial time $-4.0 \mathrm{~min}$ ), gas - helium (flow rate: $1 \mathrm{~mL} / \mathrm{min}$ ).

Identification: The content compounds were identified by comparison with standards, retention times, retention indices, and the spectral matching of libraries NIST/EPA/NIH Mass Spectral Library 2017, Wiley Registry of Mass Spectral Data 11th Edition, FFNSC $3,{ }^{\circ} 2015$, and Adams EO library, Mass Spectral Library, 2205 compounds. 


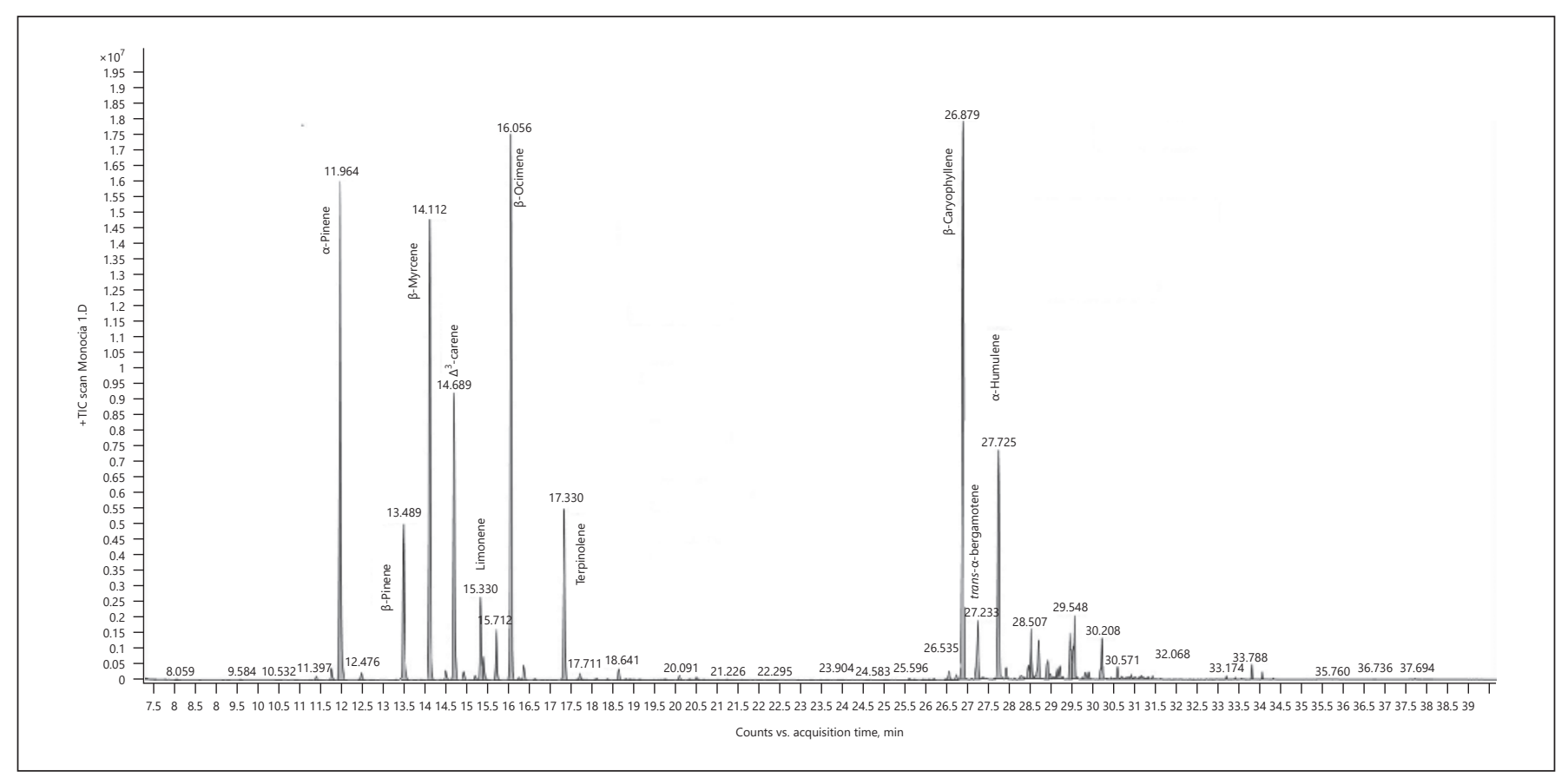

Fig. 2. Gas chromatogram of Monoica hemp chemotype.

Table 5.

\begin{tabular}{rlr}
\hline Normalized \% & Compound & RI \\
\hline 80.24 & $\boldsymbol{\alpha}$-Pinene & 937 \\
24.50 & $\boldsymbol{\beta}$-Pinene & 979 \\
71.40 & $\boldsymbol{\beta}$-Myrcene & 991 \\
45.96 & $\boldsymbol{\Delta}$-Carene & 1,011 \\
12.81 & Limonene & 1,031 \\
7.20 & $\boldsymbol{\alpha}$-Ocimene & 1,039 \\
83.29 & $\boldsymbol{\beta}$-Ocimene & 1,037 \\
27.00 & Terpinolene & 1,088 \\
$\mathbf{1 0 0 . 0 0}$ & B-Caryophyllene & 1,418 \\
8.49 & Trans-a-bergamotene & 1,435 \\
36.75 & a-Humulene & 1,454 \\
7.14 & $\boldsymbol{\beta}$-Selinene & 1,486 \\
7.09 & a-Selinene & 1,494 \\
7.86 & Selina-3,7(11)-diene & 1,542 \\
6.02 & Caryophyllene oxide & 1,581 \\
\hline
\end{tabular}

\section{Results}

In the text, every time for each mentioned chemotype, the first is a picture of their gas chromatographic/mass spectrometric analysis and after that a table of terpenes/ terpenoids.

\section{Comparison of Hemp and Cannabis}

Initially, we tried to compare the so-called hemp with the so-called cannabis, which is necessary to explain here. Hemp is a cannabis cultivated for fiber but it is also used for treatment. In fact, it is C. sativa L. with very low concentrations of CBD and THC. Cannabis is called today C. sativa L. mainly used for recreational and/or medicinal use. It has a high concentration of CBD and/or THC. The difference between recreational and medicinal cannabis is in cultivation - medicinal cannabis is cultivated under strict conditions. In fact, many chemotypes developed for recreational use are currently called medicinal cannabis. As we did not have all terpenes standards that exist in this plant, we could not quantify all terpenes and terpenoids; therefore, we used comparison based on relative ratio of the main terpene/terpenoid in the particular chemotype.

Hemp (cannabis for fibers) - chemotype Monoica (listed volatiles that are present at a level more than $5 \%$ of the main terpene - monoterpenes are marked in bold) (Fig. 2; Table 5):

Cannabis (cannabis for treatment) - chemotype Lemon OG Kush (listed volatiles that are present at a level more than $5 \%$ of the main terpene - sesquiterpenes/sesquiterpenoids are marked in bold) (Fig. 3; Table 6): 


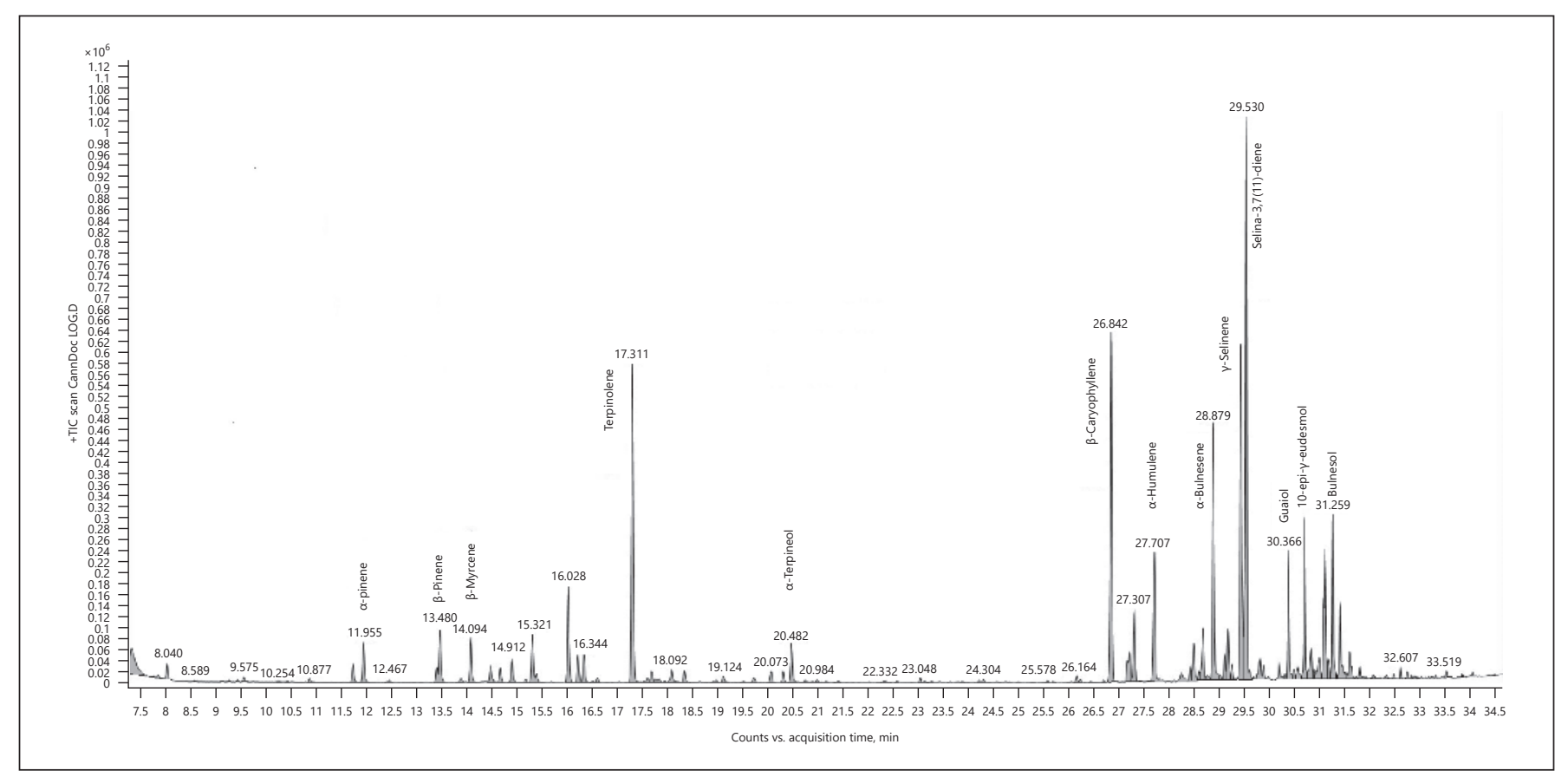

Fig. 3. Gas chromatogram of Lemon OG Kush cannabis chemotype.

\section{Results of 108 Inflorescences and Essential Oils}

Analyses

Fifty-four inflorescences of different cannabis chemotypes (Fuji, Everest, Golan, Tropicana, Kilimanjaro (CannDoc), Marom, Edom, Choco 1, Durban Poison, Jack Herer, Black Diamond, Holy Weed, Pandora’s Box, Tel Aviv, Paris, OG Kush, Bubble Gum, Strawberry 2.0, Lemon Deluxe, Berry Deluxe, Medi Kush, Lemon K2, Blue Haze, Annapurna, Choco 2, Himalaya, Lemon OG Kush, AK-47, Jericho, Chocolope, Doblin, K, Kira Kush, Kilimanjaro (Better), Luli Kush, Maui Waui, White Widow, Topaz, DOV, Alaska, Avidekel, Barak, El-Na, Erez, Or, Tal, Jasmine, Shira, Rafael, Mango, Candy Kush, Local Afgan, Power Plant, and Toffy) +9 essential oils of different hemp chemotypes (Futura, Felina, Fedora, Ferimon, Monoica, Tiborszallasi, Tisza, Bialobrzeskie from Slovenia, and Bialobrzeskie from Czech Republic) +45 essential oils of different cannabis chemotypes (Jamaican dream, Girl Scout Cookies, Lemon Cookies, Tangie, Gelato, Chocolate Mint OG, Key Lime Pie, Gorilla Glue, Pink Plant, 24K Gold, Critical Jack, Sweet Tooth, Sugaree, Kashmir Kush, Holy Grail Kush, Chemdawg 4, 3 Kings, Sour Diesel, TNT Kush, Monster, Sensi Star, SFV OG, Veneno, Gipsy Haze, Critical, 707 Truthband, Blackberry Kush, Grapefruit OG, Furious Candy, AK-47, High Level, Cinderella 99, Black Dream, 10K Jack,
Table 6.

\begin{tabular}{rlr}
\hline Normalized \% & Compound & RI \\
\hline 8.46 & a-Pinene & 937 \\
11.26 & $\beta$-Pinene & 979 \\
9.28 & $\beta$-Myrcene & 991 \\
68.01 & Terpinolene & 1,088 \\
8.85 & a-Terpineol & 1,189 \\
73.09 & $\boldsymbol{\beta}$-Caryophyllene & 1,418 \\
15.09 & $\boldsymbol{\alpha}$-Guaiene & 1,439 \\
27.01 & a-Humulene & 1,454 \\
11.06 & $\boldsymbol{\alpha}$-Sselinene & 1,494 \\
49.72 & $\boldsymbol{\alpha}$-Bulnesene & 1,505 \\
68.46 & Selina-4(15),7(11)-diene & 1,544 \\
$\mathbf{1 0 0 . 0 0}$ & Selina-3,7(11)-diene & 1,542 \\
18.95 & Guaiol & 1,596 \\
25.92 & $\mathbf{1 0}$-Epi- $\boldsymbol{\gamma}$-eudesmol & 1,619 \\
7.86 & $\boldsymbol{\gamma}$-Eudesmol & 1,631 \\
9.36 & $\boldsymbol{\beta}$-Eudesmol & 1,649 \\
19.57 & $\boldsymbol{\alpha}$-Eudesmol & 1,653 \\
25.17 & Bulnesol & 1,666 \\
15.22 & $\boldsymbol{\alpha}$-Bisabolol & 1,683 \\
\hline
\end{tabular}

Cheese, Lavender, Truth, Orange Turbo, Dosidos, Nina Limone, Amnesia, Wifi OG, M.I.B., OG Kush, Mojito) = altogether 108 chemotypes. 
Table 7.

\begin{tabular}{|c|c|c|}
\hline Compound & RI & $\begin{array}{l}\text { Between } 10 \text { mains in a sample } \\
\text { (the main in a sample) }\end{array}$ \\
\hline$\beta$-Caryophyllene & 1,418 & $108 \times(48 \times)$ \\
\hline$\beta$-Myrcene & 991 & $89 \times(19 \times)$ \\
\hline a-Pinene & 939 & $83 \times(9 \times)$ \\
\hline Limonene & 1,031 & $79 \times(2 \times)$ \\
\hline$\beta$-Pinene & 980 & $72 \times(0 \times)$ \\
\hline Linalool & 1,098 & $45 \times(1 \times)$ \\
\hline Guaiol & 1,596 & $35 \times(0 \times)$ \\
\hline 10-Epi- $\gamma$-eudesmol & 1,619 & $34 \times(5 \times)$ \\
\hline Selina-3,7(11)-diene & 1,542 & $33 \times(9 \times)$ \\
\hline a-Humulene & 1,454 & $82 \times(0 \times)$ \\
\hline Bulnesol & 1,667 & $32 \times(1 \times)$ \\
\hline$\alpha$-Terpineol & 1,189 & $28 \times(0 \times)$ \\
\hline a-Eudesmol & 1,653 & $26 \times(1 \times)$ \\
\hline$p$-Mentha-1(7),8-diene & 1,004 & $26 \times(0 \times)$ \\
\hline$\beta$-Eudesmol & 1,649 & $23 \times(1 \times)$ \\
\hline Endo-fenchol & 1,112 & $22 \times(0 \times)$ \\
\hline a-Bisabolol & 1,683 & $20 \times(3 \times)$ \\
\hline$\gamma$-Selinene & 1,544 & $20 \times(1 \times)$ \\
\hline Trans- $\beta$-ocimene & 1,044 & $20 \times(0 \times)$ \\
\hline Terpinolene & 1,088 & $18 \times(8 \times)$ \\
\hline$\alpha$-Selinene & 1,494 & $12 \times(0 \times)$ \\
\hline$\beta$-Selinene & 1,486 & $11 \times(0 \times)$ \\
\hline p-Cymene & 1,026 & $10 \times(0 \times)$ \\
\hline$\gamma$-Eudesmol & 1,631 & $9 \times(0 \times)$ \\
\hline a-Bulnesene & 1,505 & $9 \times(0 \times)$ \\
\hline$\gamma$-Elemene & 1,434 & $9 \times(0 \times)$ \\
\hline Camphene & 953 & $9 \times(0 \times)$ \\
\hline Germacrene B & 1,557 & $8 \times(0 \times)$ \\
\hline a-Guaiene & 1,439 & $4 \times(0 \times)$ \\
\hline Epi-a-bisabolol & 1,670 & $4 \times(0 \times)$ \\
\hline Juniper camphor & 1,692 & $3 \times(0 \times)$ \\
\hline Cis- $\alpha$-bisabolene & 1,504 & $3 \times(0 \times)$ \\
\hline Trans- $\alpha$-bisabolene & 1,512 & $3 \times(0 \times)$ \\
\hline Trans-a-bergamotene & 1,435 & $9 \times(0 \times)$ \\
\hline$\Delta^{3}$-Carene & 1,011 & $7 \times(0 \times)$ \\
\hline Caryophyllene oxide & 1,581 & $7 \times(0 \times)$ \\
\hline 1,8-Cineole & 1,032 & $6 \times(0 \times)$ \\
\hline a-Ocimene & 1,039 & $5 \times(0 \times)$ \\
\hline Hexyl butyrate & 1,192 & $5 \times(0 \times)$ \\
\hline Cis- $\beta$-ocimene & 1,040 & $4 \times(0 \times)$ \\
\hline a-Phellandrene & 1,005 & $4 \times(0 \times)$ \\
\hline Borneol & 1,166 & $4 \times(0 \times)$ \\
\hline$\beta$-Phellandrene & 1,031 & $4 \times(0 \times)$ \\
\hline$\alpha$-Terpinene & 1,017 & $3 \times(0 \times)$ \\
\hline$\delta$-Selinene & 1,493 & $2 \times(0 \times)$ \\
\hline Trans- $\beta$-farnesene & 1,458 & $2 \times(0 \times)$ \\
\hline Trans-nerolidol & 1,564 & $2 \times(0 \times)$ \\
\hline$\gamma$-Terpinene & 1,060 & $2 \times(0 \times)$ \\
\hline Trans-pinene hydrate & 1,140 & $2 \times(0 \times)$ \\
\hline Ocimene & 1,050 & $1 \times(0 \times)$ \\
\hline Exo-fenchol & 1,116 & $1 \times(0 \times)$ \\
\hline Cis-pinene hydrate & 1,121 & $1 \times(0 \times)$ \\
\hline Trans- $\beta$-guaiene & 1,500 & $1 \times(0 \times)$ \\
\hline$\gamma$-Cadinene & 1,513 & $1 \times(0 \times)$ \\
\hline$\beta$-Curcumene & 1,514 & $1 \times(0 \times)$ \\
\hline Selin-6-en-4a-ol & 1,636 & $1 \times(0 \times)$ \\
\hline Humulene epoxide I & 1,594 & $2 \times(0 \times)$ \\
\hline$\gamma$-Terpineol & 1,197 & $1 \times(0 \times)$ \\
\hline
\end{tabular}

The bold values represent the maximum amount when the compound was between the ten main terpenes.
Table 8.

\begin{tabular}{|c|c|c|}
\hline Compound & RI & $\begin{array}{l}\text { Between } 10 \text { mains } \\
\text { in a sample } \\
\text { (the main in a sample) }\end{array}$ \\
\hline$\beta$-Caryophyllene & 1,418 & $53 \times(13 \times)$ \\
\hline$\beta$-Myrcene & 991 & $36 \times(13 \times)$ \\
\hline Guaiol & 1,596 & $35 \times(0 \times)$ \\
\hline 10 -Epi- $\gamma$-eudesmol & 1,619 & $34 \times(5 \times)$ \\
\hline Selina-3,7(11)-diene & 1,542 & $33 \times(9 \times)$ \\
\hline a-Humulene & 1,454 & $36 \times(0 \times)$ \\
\hline Bulnesol & 1,667 & $32 \times(1 \times)$ \\
\hline a-Pinene & 939 & $29 \times(5 \times)$ \\
\hline a-Eudesmol & 1,653 & $26 \times(1 \times)$ \\
\hline Limonene & 1,031 & $27 \times(0 \times)$ \\
\hline$\beta$-Eudesmol & 1,649 & $23 \times(1 \times)$ \\
\hline a-Bisabolol & 1,683 & $20 \times(3 \times)$ \\
\hline$\gamma$-Selinene & 1,544 & $20 \times(1 \times)$ \\
\hline$\beta$-Pinene & 980 & $18 \times(0 \times)$ \\
\hline a-Selinene & 1,494 & $11 \times(0 \times)$ \\
\hline Terpinolene & 1,088 & $9 \times(2 \times)$ \\
\hline$\gamma$-Eudesmol & 1,631 & $9 \times(0 \times)$ \\
\hline a-Bulnesene & 1,505 & $9 \times(0 \times)$ \\
\hline Linalool & 1,098 & $9 \times(0 \times)$ \\
\hline$\gamma$-Elemene & 1,434 & $9 \times(0 \times)$ \\
\hline Germacrene B & 1,557 & $8 \times(0 \times)$ \\
\hline$\beta$-Selinene & 1,486 & $8 \times(0 \times)$ \\
\hline Endo-fenchol & 1,112 & $6 \times(0 \times)$ \\
\hline a-Guaiene & 1,439 & $4 \times(0 \times)$ \\
\hline Epi-a-bisabolol & 1,670 & $4 \times(0 \times)$ \\
\hline Juniper camphor & 1,692 & $3 \times(0 \times)$ \\
\hline Cis- $a$-bisabolene & 1,504 & $3 \times(0 \times)$ \\
\hline Trans-a-bisabolene & 1,512 & $3 \times(0 \times)$ \\
\hline a-Terpineol & 1,189 & $3 \times(0 \times)$ \\
\hline Trans- $\beta$-ocimene & 1,044 & $3 \times(0 \times)$ \\
\hline$\beta$-Phellandrene & 1,031 & $2 \times(0 \times)$ \\
\hline Trans-a-bergamotene & 1,435 & $2 \times(0 \times)$ \\
\hline$\delta$-Selinene & 1,493 & $2 \times(0 \times)$ \\
\hline Ocimene & 1,050 & $1 \times(0 \times)$ \\
\hline Exo-fenchol & 1,116 & $1 \times(0 \times)$ \\
\hline Cis-pinene hydrate & 1,121 & $1 \times(0 \times)$ \\
\hline Trans-pinene hydrate & 1,140 & $1 \times(0 \times)$ \\
\hline Trans- $\beta$-farnesene & 1,458 & $2 \times(0 \times)$ \\
\hline Trans- $\beta$-guaiene & 1,500 & $1 \times(0 \times)$ \\
\hline$\gamma$-Cadinene & 1,513 & $1 \times(0 \times)$ \\
\hline$\beta$-Curcumene & 1,514 & $1 \times(0 \times)$ \\
\hline Trans-nerolidol & 1,564 & $1 \times(0 \times)$ \\
\hline Caryophyllene oxide & 1,581 & $1 \times(0 \times)$ \\
\hline Selin-6-en-4a-ol & 1,636 & $1 \times(0 \times)$ \\
\hline
\end{tabular}

The bold values represent the maximum amount when the compound was between the ten main terpenes. 
Table 9.

\begin{tabular}{|c|c|c|}
\hline Compound & RI & $\begin{array}{l}\text { Between } 10 \text { mains } \\
\text { in a sample } \\
\text { (the main in a sample) }\end{array}$ \\
\hline$\beta$-Caryophyllene & 1,418 & $46 \times(30 \times)$ \\
\hline a-Pinene & 937 & $45 \times(4 x)$ \\
\hline$\beta$-Myrcene & 991 & $45 \times(3 \times)$ \\
\hline Limonene & 1,031 & $45 \times(2 \times)$ \\
\hline$\beta$-Pinene & 979 & $45 \times(0 \times)$ \\
\hline a-Humulene & 1,454 & $37 \times(0 \times)$ \\
\hline Linalool & 1,098 & $36 \times(1 \times)$ \\
\hline$p$-Mentha-1(7),8-diene & 1,004 & $26(0 \times)$ \\
\hline a-Terpineol & 1,189 & $25 \times(0 \times)$ \\
\hline Terpinolene & 1,088 & $21 \times(6 \times)$ \\
\hline Endo-fenchol & 1,112 & $16 \times(0 \times)$ \\
\hline$\beta$-Ocimene & 1,040 & $13 \times(0 \times)$ \\
\hline p-Cymene & 1,026 & $10 \times(0 \times)$ \\
\hline Camphene & 953 & $9 \times(0 \times)$ \\
\hline 1,8-Cineole & 1,032 & $6 \times(0 \times)$ \\
\hline Hexyl butyrate & 1,192 & $5 \times(0 \times)$ \\
\hline a-Phellandrene & 1,005 & $4 \times(0 \times)$ \\
\hline$\Delta^{3}$-Carene & 1,011 & $4 \times(0 \times)$ \\
\hline a-Ocimene & 1,039 & $4 \times(0 \times)$ \\
\hline Borneol & 1,166 & $4 \times(0 \times)$ \\
\hline Caryophyllene oxide & 1,581 & $4 \times(0 \times)$ \\
\hline a-Terpinene & 1,017 & $3 \times(0 \times)$ \\
\hline Trans- $\beta$-ocimene & 1,049 & $2 \times(0 \times)$ \\
\hline$\gamma$-Terpinene & 1,060 & $2 \times(0 \times)$ \\
\hline Trans-pinene hydrate & 1,140 & $1 \times(0 \times)$ \\
\hline$\gamma$-Terpineol & 1,197 & $1 \times(0 \times)$ \\
\hline Trans-nerolidol & 1,564 & $1 \times(0 \times)$ \\
\hline
\end{tabular}

The bold values represent the maximum amount when the compound was between the ten main terpenes.

a. Fifty-eight different terpenes were found between the 10 main terpenes from each of 108 chemotypes (hemp and cannabis inflorescence and essential oil samples).

Sorting according to the frequency of the main terpene in the 10 main ones at each chemotype (numbers outside parentheses indicate in how many phenotypes was this particular terpene between the 10 main ones, and numbers in parentheses indicate in how many phenotypes was this particular terpene/terpenoid the major one) (Table 7).

b. Forty-four different terpenes were found between the 10 main terpenes of each from 54 chemotypes (cannabis inflorescence samples).

Sorting according to the frequency of the main terpene in the 10 main ones at each chemotype (numbers outside parentheses indicates in how many phenotypes was this particular terpene between the 10 main ones, and num-
Table 10.

\begin{tabular}{|c|c|c|}
\hline Compound & RI & $\begin{array}{l}\text { Between } 10 \text { mains } \\
\text { in a sample } \\
\text { (the main in a sample) }\end{array}$ \\
\hline$\beta$-Caryophyllene & 1,418 & $8 \times(5 \times)$ \\
\hline$\beta$-Myrcene & 991 & $7 \times(4 \times)$ \\
\hline a-Pinene & 937 & $8 \times(0 \times)$ \\
\hline$\beta$-Pinene & 979 & $8 \times(0 \times)$ \\
\hline Terpinolene & 1,088 & $8 \times(0 \times)$ \\
\hline a-Humulene & 1,454 & $8 \times(0 \times)$ \\
\hline Limonene & 1,031 & $7 \times(0 \times)$ \\
\hline$\beta$-Ocimene & 1,037 & $6 \times(0 \times)$ \\
\hline Trans-a-bergamotene & 1,435 & $6 \times(0 \times)$ \\
\hline$\Delta^{3}$-Carene & 1,011 & $3 \times(0 \times)$ \\
\hline$\beta$-Selinene & 1,486 & $3 \times(0 \times)$ \\
\hline Cis- $\beta$-ocimene & 1,040 & $2 \times(0 \times)$ \\
\hline Caryophyllene oxide & 1,581 & $2 \times(0 \times)$ \\
\hline$\beta$-Phellandrene & 1,031 & $1 \times(0 \times)$ \\
\hline a-Ocimene & 1,039 & $1 \times(0 \times)$ \\
\hline a-Selinene & 1,494 & $1 \times(0 \times)$ \\
\hline Humulene epoxide I & 1,594 & $1 \times(0 \times)$ \\
\hline
\end{tabular}

The bold values represent the maximum amount when the compound was between the ten main terpenes.

bers in parentheses indicate in how many phenotypes was this particular terpene the major one) (Table 8).

c. Twenty-seven different terpenes were found between the 10 main terpenes of each from 46 chemotypes (cannabis essential oils).

Sorting according to the frequency of the main terpene in the 10 main ones at each chemotype (numbers outside parentheses indicate in how many phenotypes was this particular terpene between the 10 main ones, and numbers in parentheses indicates in how many phenotypes was this particular terpene the major one) (Table 9).

d. Seventeen different terpenes were found between the 10 main terpenes of each from 7 chemotypes (hemp essential oils).

Sorting according to the frequency of the main terpene in the 10 main ones at each chemotype (numbers outside parentheses indicate in how many phenotypes was this particular terpene between the 10 main ones, and numbers in parentheses indicate in how many phenotypes was this particular terpene the major one) (Table 10).

e. Examples of different chemotypes with different main terpenes:

1. a-Pinene-dominant chemotype - Kilimanjaro (Fig. 4; Table 11)

2. $\beta$-Myrcene dominant chemotype - Durban Poison (Fig. 5; Table 12) 


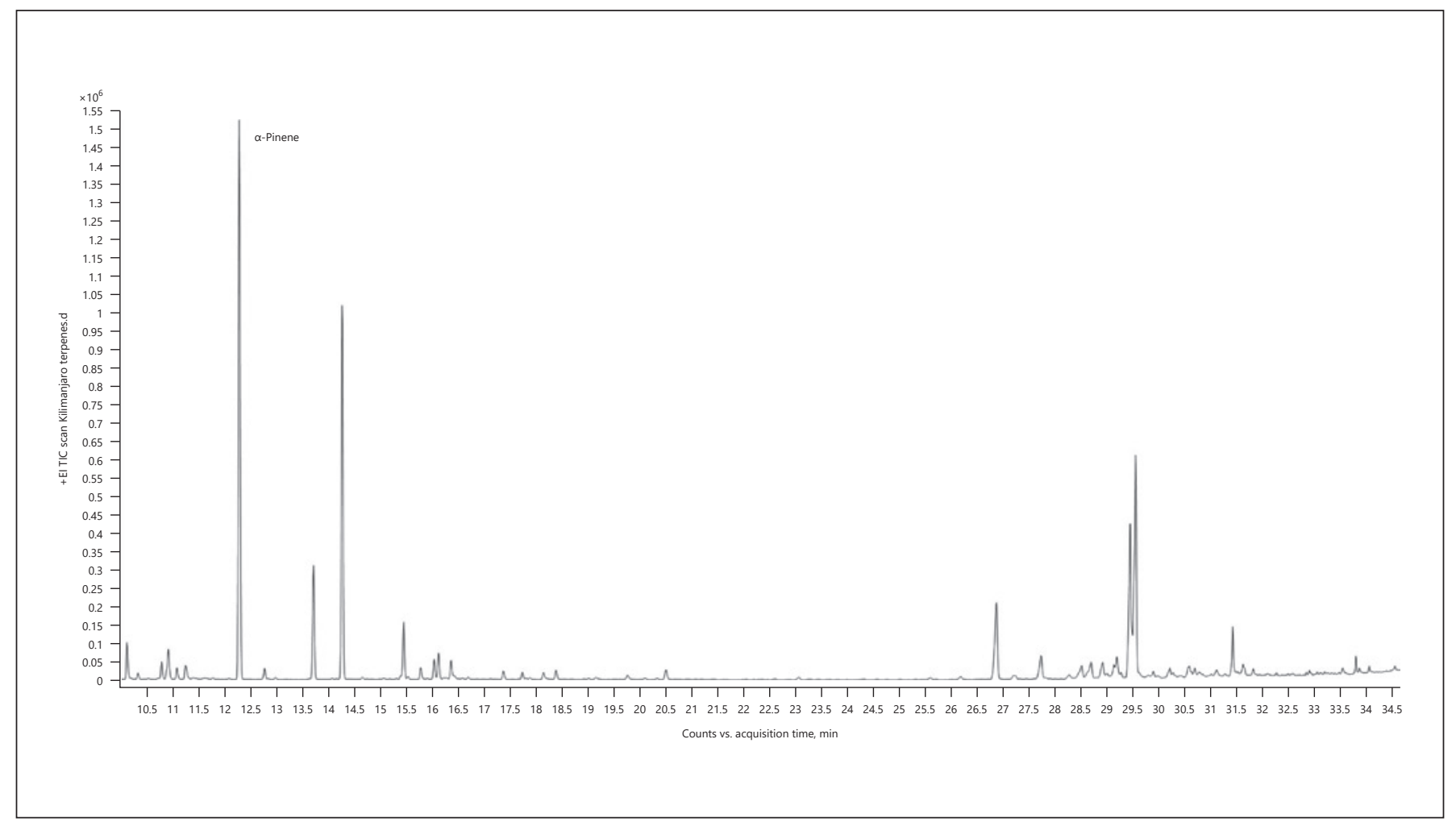

Fig. 4. Gas chromatogram of Kilimanjaro cannabis chemotype.

Table 11.

\begin{tabular}{clr}
\hline Normalized \% & Compound & RI \\
\hline $\mathbf{1 0 0 . 0 0}$ & a-Pinene & 939 \\
64.84 & $\beta$-Myrcene & 991 \\
52.98 & - & 1,544 \\
39.58 & Selinene & 1,542 \\
22.90 & Caryophyllene & 1,418 \\
21.12 & B-Pinene & 980 \\
11.23 & Limonene & 1,031 \\
8.70 & a-Bisabolol & 1,683 \\
5.70 & a-Selinene & 1,494 \\
4.93 & Ocimene & 1,050 \\
\hline
\end{tabular}

The bold value represents the main compound in the sample.
3. Limonene-dominant chemotype - Sweet Tooth (Fig. 6; Table 13)

4. Terpinolene-dominant chemotype - Jack Herer (Fig. 7; Table 14)

5. Linalool-dominant chemotype - Dosidos (Fig. 8; Table 15)

6. $\beta$-Caryophyllene-dominant chemotype - Edom (Fig. 9; Table 16)

7. Selina-3,7(11)-diene-dominant chemotype - Lemon OG Kush (Fig. 10; Table 17)

8. $\gamma$-Selinene-dominant chemotype - Fuji (Fig. 11; Table 18)

9. 10-Epi- $\gamma$-eudesmol-dominant chemotype - Lemon Deluxe (Fig. 12; Table 19)

10. $\beta$-Eudesmol-dominant chemotype - Alaska (Fig. 13; Table 20)

11.a-Eudesmol-dominant chemotype - El Na (Fig. 14; Table 21)

12.Bulnesol-dominant chemotype - Berry Deluxe (Fig. 15; Table 22)

13.a-Bisabolol-dominant chemotype - Bubble Gum (Fig. 16; Table 23) 


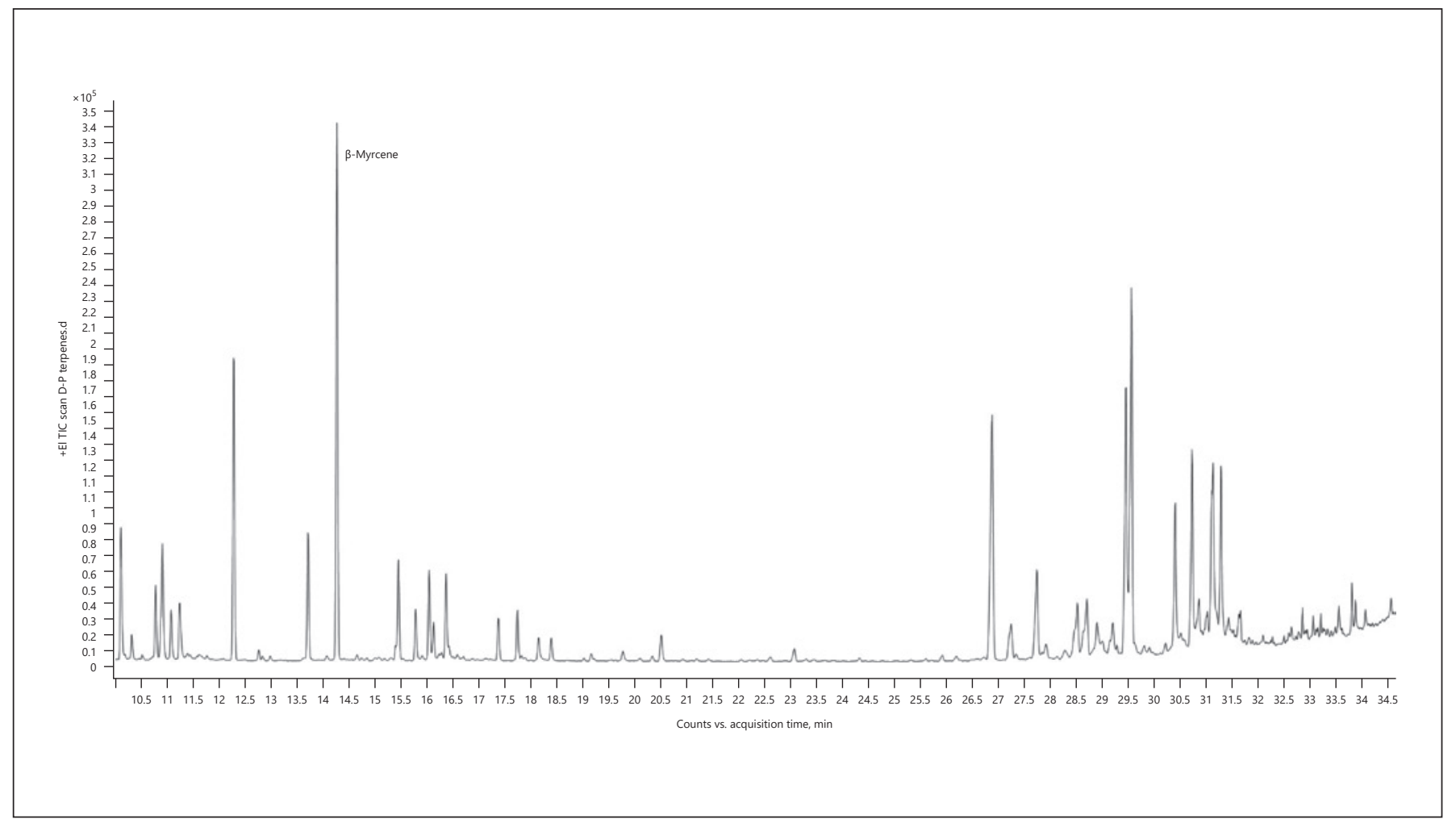

Fig. 5. Gas chromatogram of Durban Poison cannabis chemotype.

Table 12.

\begin{tabular}{|c|c|c|}
\hline Normalized \% & Compound & RI \\
\hline 100.00 & $\beta$-Myrcene & 991 \\
\hline 79.20 & Caryophyllene & 1,418 \\
\hline 61.69 & a-Pinene & 939 \\
\hline 47.22 & Guaiol & 1,596 \\
\hline 46.16 & $\beta$-Eudesmol & 1,649 \\
\hline 36.21 & a-Eudesmol & 1,652 \\
\hline 31.69 & Selina-3,7(11)-diene & 1,542 \\
\hline 28.87 & a-Humulene & 1,440 \\
\hline 26.69 & $\beta$-Pinene & 980 \\
\hline 24.21 & Limonene & 1,031 \\
\hline
\end{tabular}

The bold value represents the main compound in the sample. 


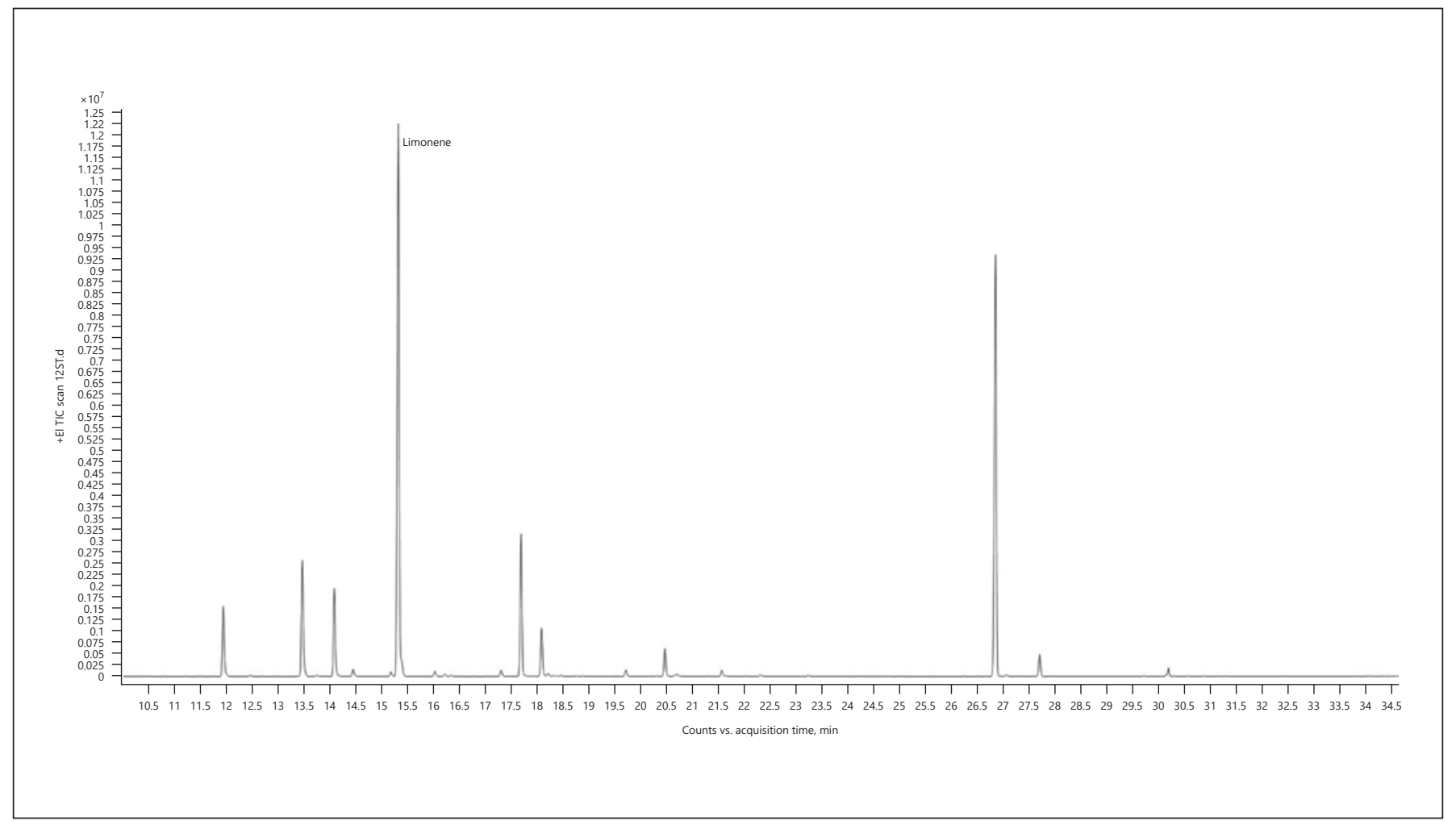

Fig. 6. Gas chromatogram of Sweet Tooth cannabis chemotype.

Table 13.

\begin{tabular}{rlr}
\hline Normalized \% & Compound & RI \\
\hline $\mathbf{1 0 0 . 0 0}$ & Limonene & 1,031 \\
76.70 & $\beta$-Caryophyllene & 1,418 \\
23.89 & Linalool & 1,098 \\
20.70 & $\beta$-Pinene & 979 \\
14.59 & $\beta$-Myrcene & 991 \\
12.49 & a-Pinene & 937 \\
8.78 & Endo-fenchol & 1,112 \\
4.90 & $\alpha$-Terpineol & 1,189 \\
3.83 & $\alpha$-Humulene & 1,454 \\
1.36 & p-Mentha-1(7),8-diene & 1,004 \\
\hline
\end{tabular}

The bold value represents the main compound in the sample. 


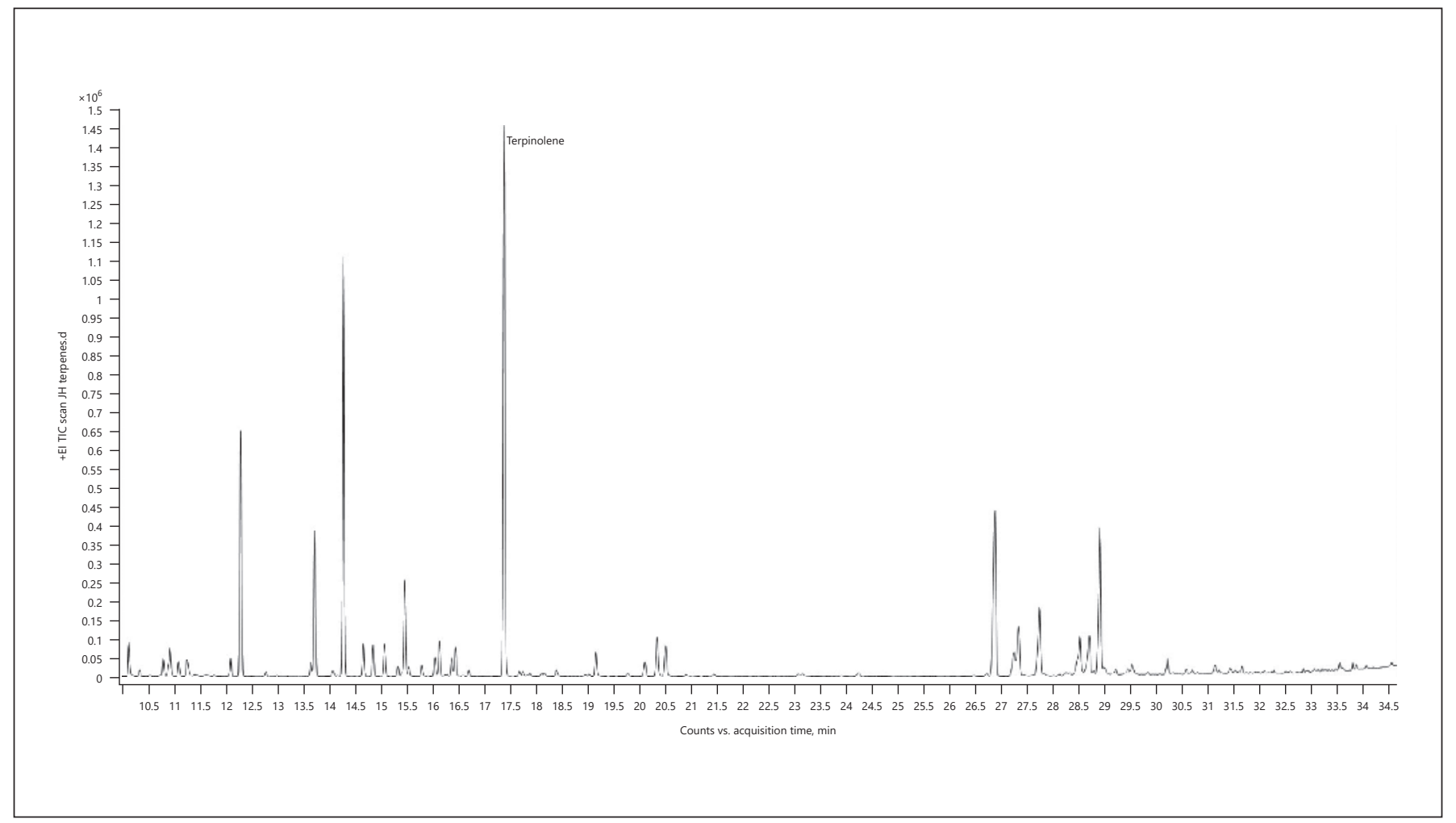

Fig. 7. Gas chromatogram of Jack Herer cannabis chemotype.

Table 14.

\begin{tabular}{clr}
\hline Normalized \% & Compound & \multicolumn{1}{c}{ RI } \\
\hline $\mathbf{1 0 0 . 0 0}$ & Terpinolene & 1,088 \\
69.43 & B-Myrcene & 991 \\
46.40 & Caryophyllene & 1,419 \\
44.23 & a-Pinene & 937 \\
34.20 & a-Bulnesene & 1,505 \\
26.69 & $\beta$-Pinene & 979 \\
19.47 & Humulene & 1,454 \\
17.98 & Limonene & 1,031 \\
13.54 & $\beta$-Selinene & 1,485 \\
13.14 & $\alpha$-Guaiene & 1,439 \\
\hline
\end{tabular}

The bold value represents the main compound in the sample. 


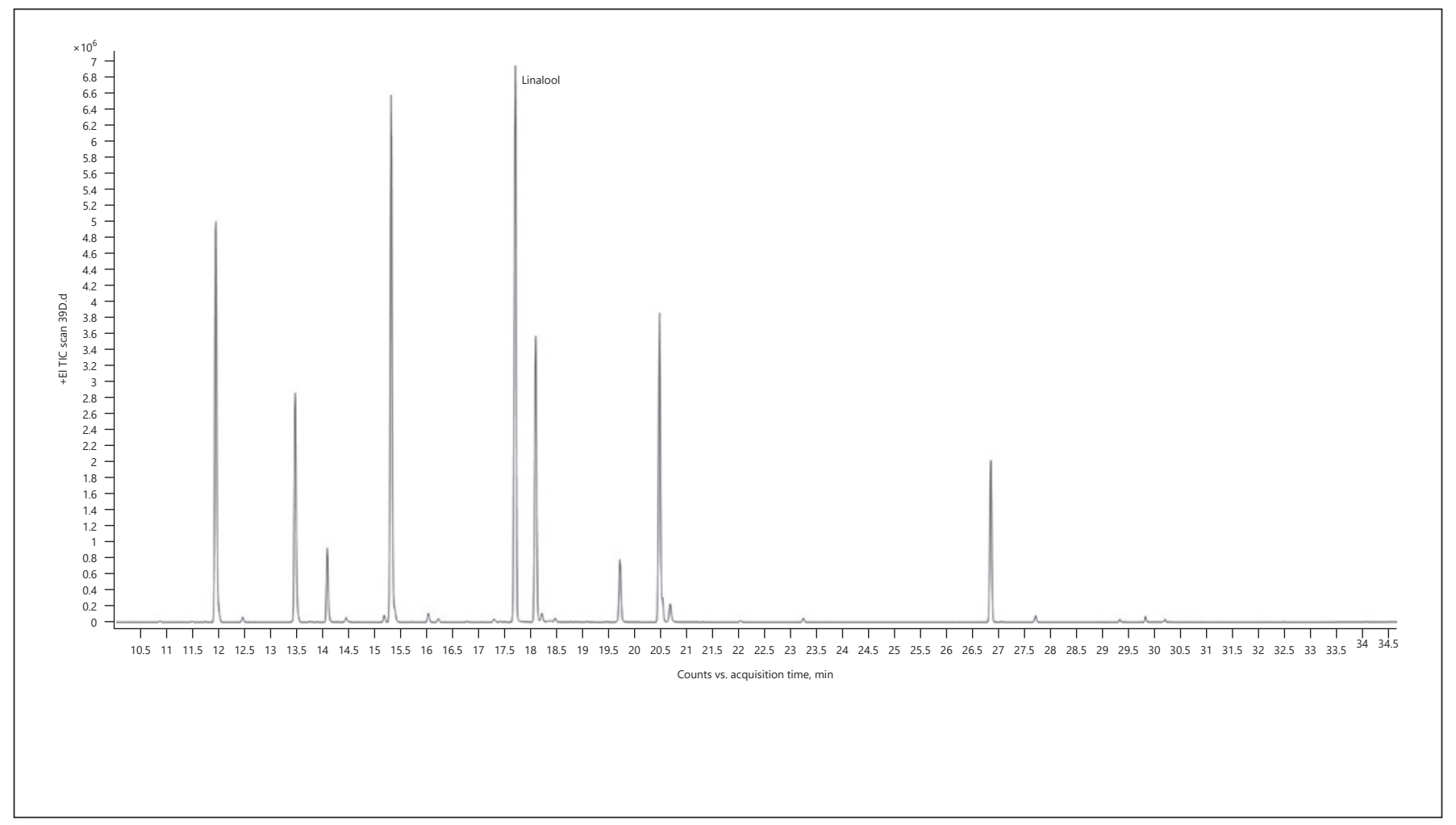

Fig. 8. Gas chromatogram of Dosidos cannabis chemotype.

Table 15.

\begin{tabular}{|c|c|c|}
\hline Normalized \% & Compound & RI \\
\hline 100.00 & Linalool & 1,098 \\
\hline 93.11 & Limonene & 1,031 \\
\hline 73.64 & a-Pinene & 937 \\
\hline 58.11 & a-Terpineol & 1,189 \\
\hline 51.17 & Endo-fenchol & 1,112 \\
\hline 41.71 & $\beta$-Pinene & 979 \\
\hline 29.27 & $\beta$-Caryophyllene & 1,418 \\
\hline 12.35 & $\beta$-Myrcene & 991 \\
\hline 11.83 & Borneol & 1,166 \\
\hline 3.87 & $\gamma$-Terpineol & 1,197 \\
\hline
\end{tabular}

The bold value represents the main compound in the sample. 


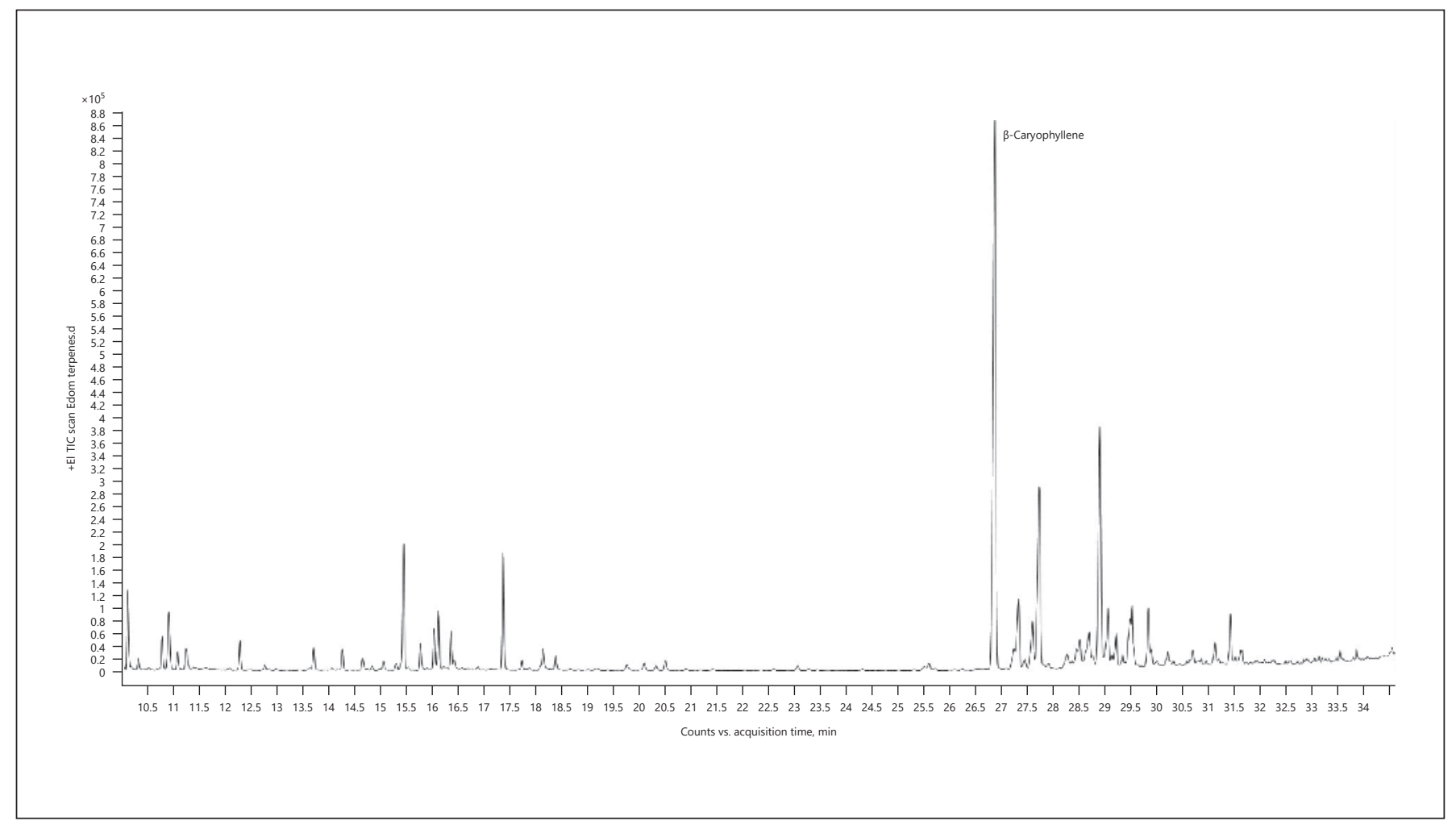

Fig. 9. Gas chromatogram of Edom cannabis chemotype.

Table 16.

\begin{tabular}{|c|c|c|}
\hline Normalized \% & Compound & RI \\
\hline 100.00 & $\beta$-Caryophyllene & 1,418 \\
\hline 40.90 & a-Bulnesene & 1,505 \\
\hline 33.50 & a-Humulene & 1,454 \\
\hline 15.05 & Limonene & 1,031 \\
\hline 14.20 & Terpinolene & 1,088 \\
\hline 12.06 & a-Guaiene & 1,439 \\
\hline 10.20 & a-Selinene & 1,494 \\
\hline 10.00 & $\gamma$-Cadinene & 1,513 \\
\hline 9.80 & Trans-nerolidol & 1,564 \\
\hline 8.40 & $\beta$-Selinene & 1,485 \\
\hline
\end{tabular}

The bold value represents the main compound in the sample. 


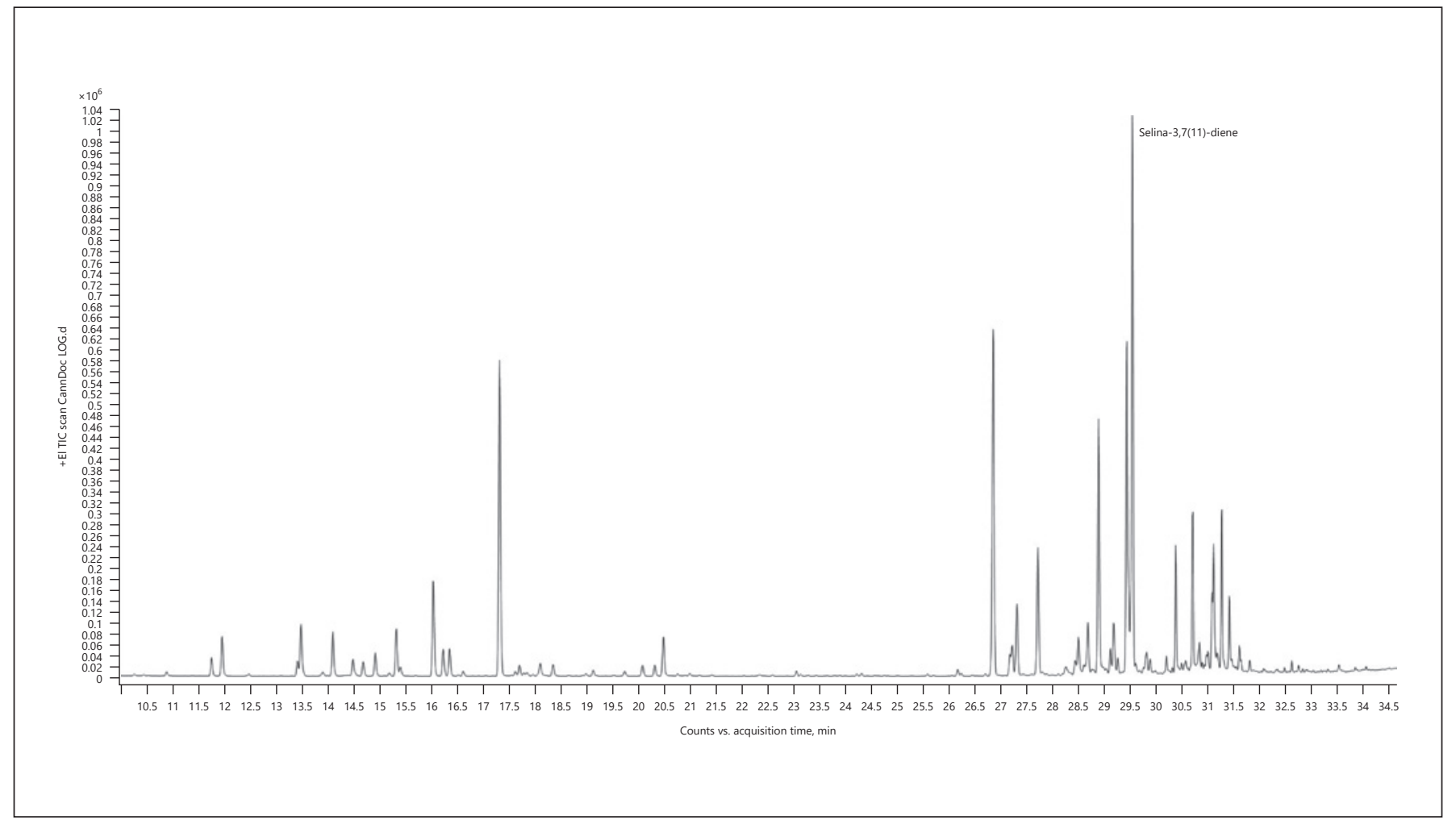

Fig. 10. Gas chromatogram of Lemon OG Kush cannabis chemotype.

Table 17.

\begin{tabular}{cll}
\hline Normalized \% & Compound & RI \\
\hline $\mathbf{1 0 0 . 0 0}$ & Selina-3,7(11)-diene & 1,542 \\
73.09 & ß-Caryophyllene & 1,418 \\
68.46 & Selina-4(15),7(11)-diene & 1,544 \\
68.01 & Terpinolene & 1,088 \\
49.72 & a-Bulnesene & 1,505 \\
27.01 & a-Humulene & 1,454 \\
25.92 & 10-Epi- $\gamma$-eudesmol & 1,619 \\
25.17 & Bulnesol & 1,666 \\
19.57 & a-Eudesmol & 1,653 \\
18.95 & Guaiol & 1,596 \\
\hline
\end{tabular}

The bold value represents the main compound in the sample. 


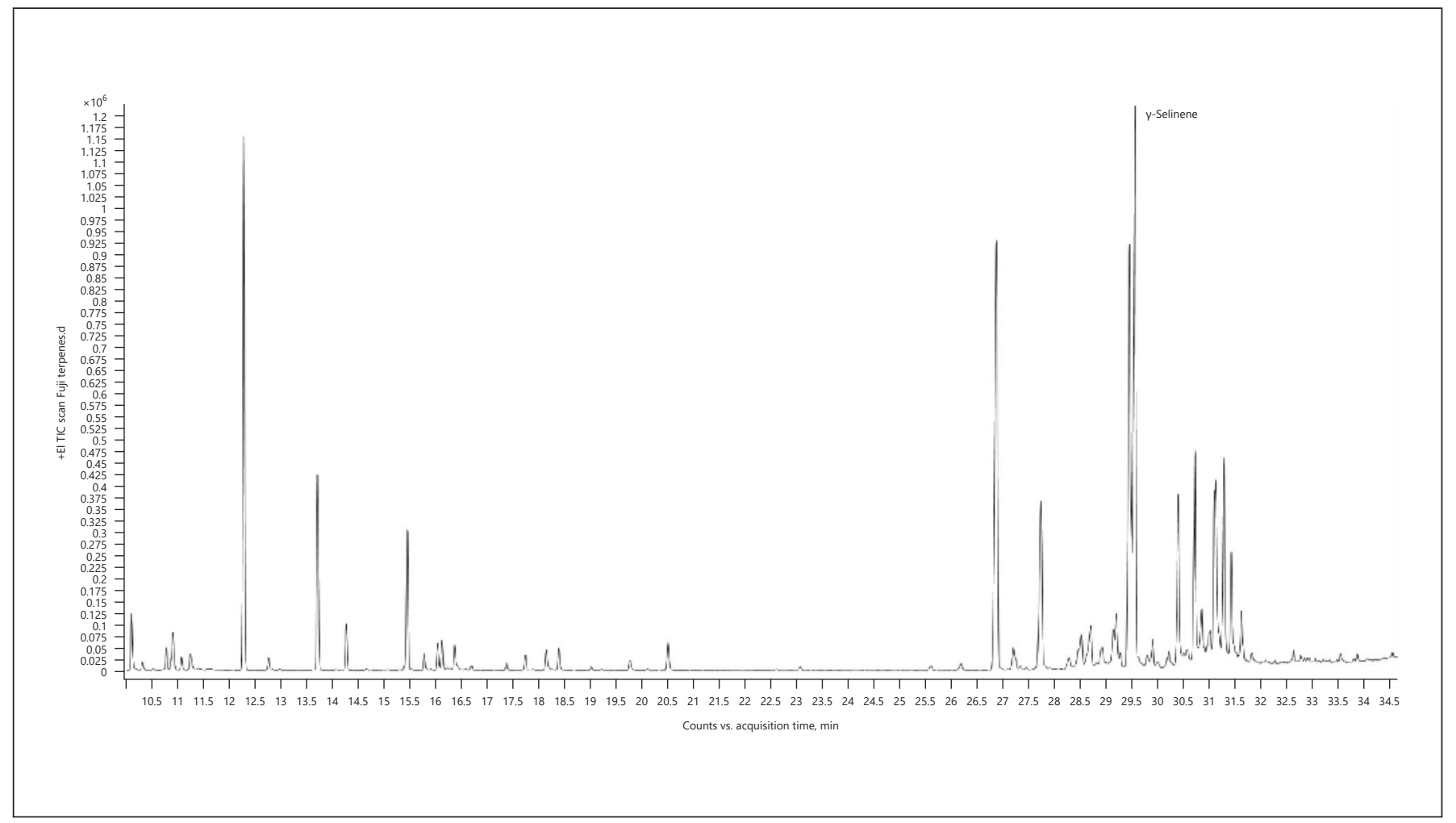

Fig. 11. Gas chromatogram of Fuji cannabis chemotype.

Table 18.

\begin{tabular}{clr}
\hline Normalized \% & Compound & RI \\
\hline $\mathbf{1 0 0 . 0 0}$ & -Selinene & 1,544 \\
94.82 & Caryophyllene & 1,418 \\
80.95 & Selina-3,7(11)-diene & 1,542 \\
72.37 & a-Pinene & 939 \\
36.95 & a-Humulene & 1,454 \\
36.18 & 10-Epi- $\gamma$-eudesmol & 1,619 \\
31.80 & Bulnesol & 1,666 \\
27.01 & Guaiol & 1,595 \\
26.82 & $\beta$-Pinene & 980 \\
23.43 & a-Eudesmol & 1,652 \\
\hline
\end{tabular}

The bold value represents the main compound in the sample. 


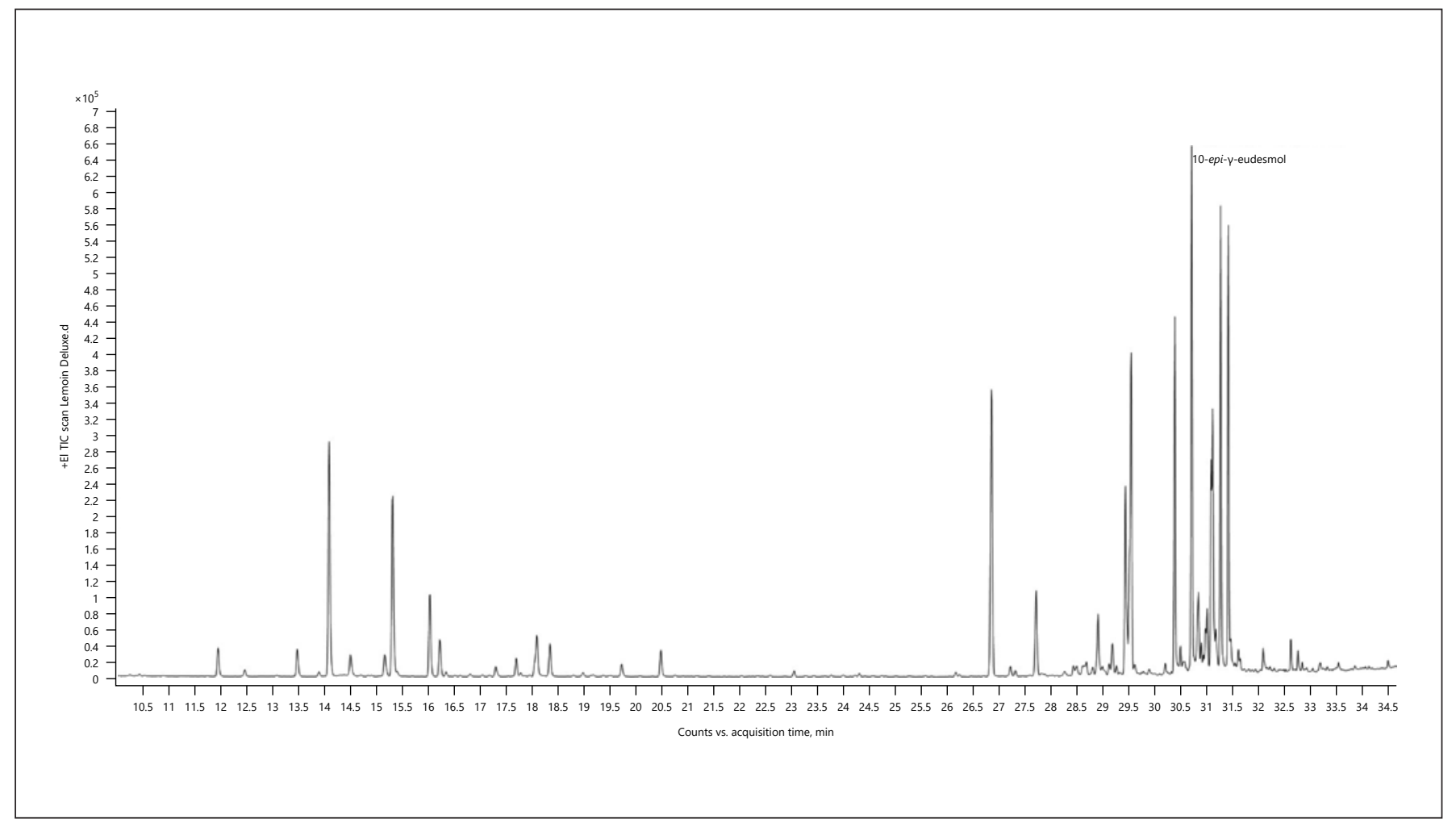

Fig. 12. Gas chromatogram of Lemon Deluxe cannabis chemotype.

Table 19.

\begin{tabular}{clr}
\hline Normalized \% & Compound & RI \\
\hline $\mathbf{1 0 0 . 0 0}$ & 10-Epi- $\boldsymbol{\gamma}$-eudesmol & 1,619 \\
93.89 & Selina-3,7(11)-diene & 1,542 \\
86.72 & a-Bisabolol & 1,683 \\
86.47 & $\beta$-Caryophyllene & 1,418 \\
83.15 & Bulnesol & 1,666 \\
68.04 & Guaiol & 1,596 \\
67.13 & $\beta$-Myrcene & 991 \\
62.23 & $\alpha$-Eudesmol & 1,653 \\
57.63 & Limonene & 1,030 \\
30.13 & $\beta$-Eudesmol & 1,649 \\
\hline
\end{tabular}

The bold value represents the main compound in the sample. 


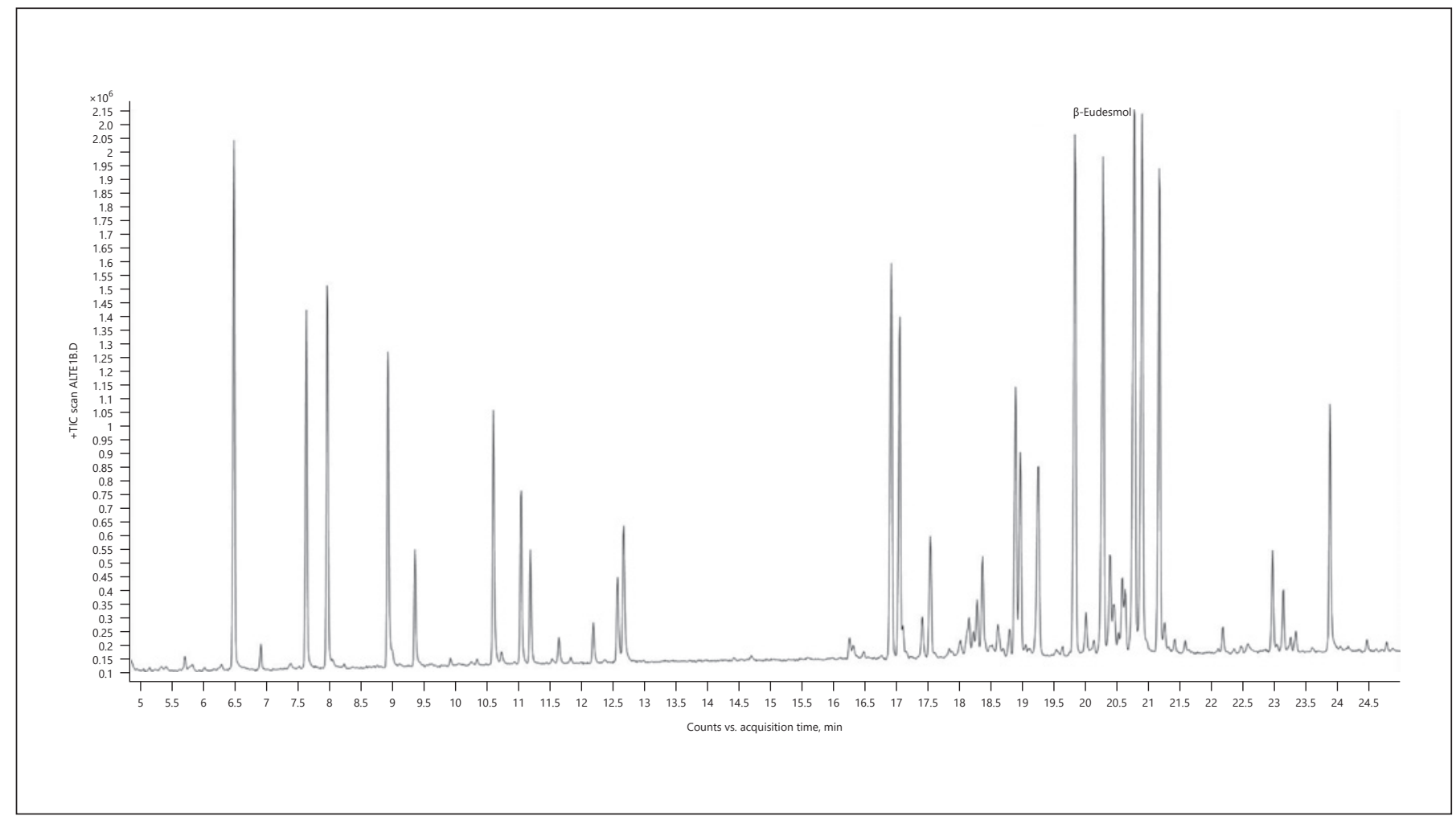

Fig. 13. Gas chromatogram of Alaska cannabis chemotype.

Table 20.

\begin{tabular}{clr}
\hline Normalized \% & Compound & RI \\
\hline $\mathbf{1 0 0 . 0 0}$ & $\boldsymbol{\beta}$-Eudesmol & 1,649 \\
87.07 & Bulnesol & 1,667 \\
81.59 & Guaiol & 1,596 \\
78.76 & 10-Epi- $\boldsymbol{\gamma}$-eudesmol & 1,619 \\
72.13 & $\alpha$-Bisabolol & 1,684 \\
65.67 & $\alpha$-Pinene & 937 \\
62.62 & $\beta$-Caryophyllene & 1,419 \\
50.71 & -Elemene & 1,434 \\
49.94 & $\beta$-Myrcene & 991 \\
44.18 & $\beta$-Pinene & 979 \\
\hline
\end{tabular}

The bold value represents the main compound in the sample. 


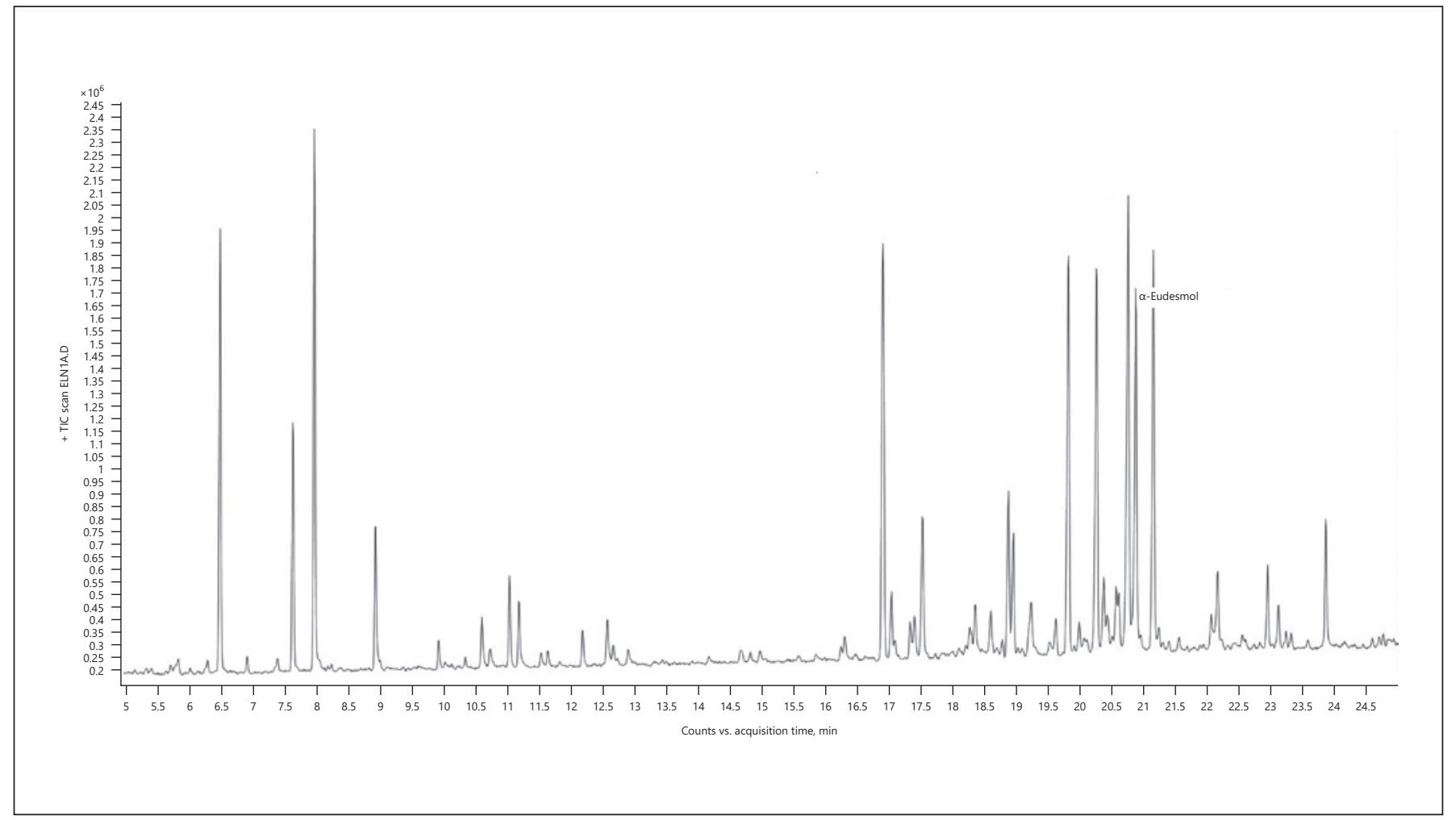

Fig. 14. Gas chromatogram of El Na cannabis chemotype.

Table 21.

\begin{tabular}{clr}
\hline Normalized \% & Compound & RI \\
\hline $\mathbf{1 0 0 . 0 0}$ & a-Eudesmol & 1,652 \\
89.80 & $\beta$-Myrcene & 991 \\
89.68 & $\beta$-Caryophyllene & 1,418 \\
82.64 & 10-Epi- $\gamma$-eudesmol & 1,619 \\
82.18 & Guaiol & 1,595 \\
79.76 & Bulnesol & 1,667 \\
74.35 & Epi-a-bisabolol & 1,686 \\
70.04 & a-Pinene & 937 \\
40.58 & $\beta$-Pinene & 979 \\
30.66 & $\alpha$-Humulene & 1,454 \\
\hline
\end{tabular}

The bold value represents the main compound in the sample. 


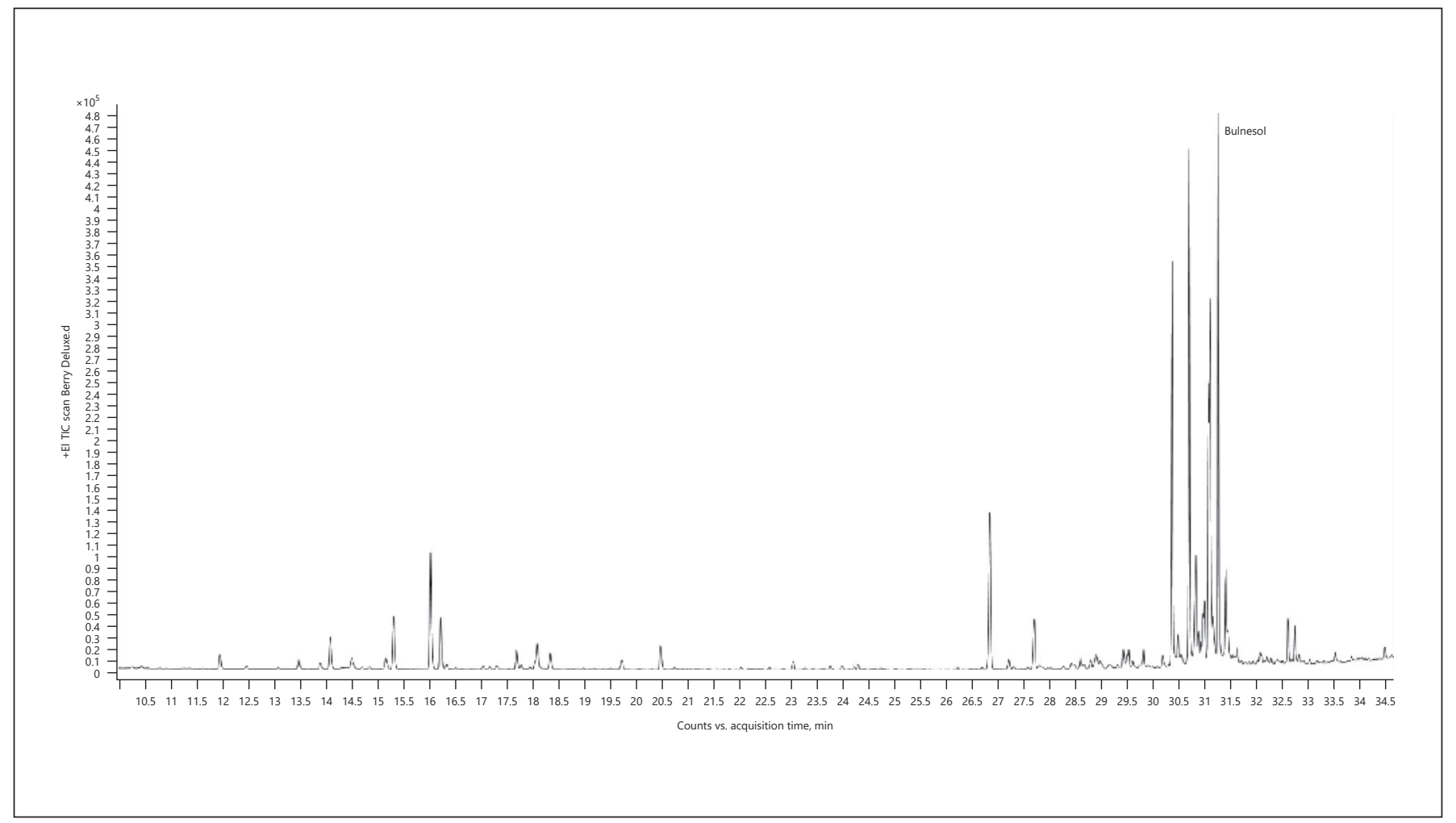

Fig. 15. Gas chromatogram of Berry Deluxe cannabis chemotype.

Table 22.

\begin{tabular}{cll}
\hline Normalized \% & Compound & RI \\
\hline $\mathbf{1 0 0 . 0 0}$ & Bulnesol & 1,666 \\
98.07 & 10-Epi- $\gamma$-eudesmol & 1,619 \\
80.32 & $\alpha$-Eudesmol & 1,653 \\
77.83 & Guaiol & 1,596 \\
46.87 & $\beta$-Caryophyllene & 1,418 \\
40.77 & $\beta$-Eudesmol & 1,649 \\
33.94 & $\alpha$-Bisabolol & 1,683 \\
32.36 & $\gamma$-Eudesmol & 1,631 \\
15.30 & Limonene & 1,030 \\
14.58 & $\alpha$-Humulene & 1,454 \\
\hline
\end{tabular}

The bold value represents the main compound in the sample. 


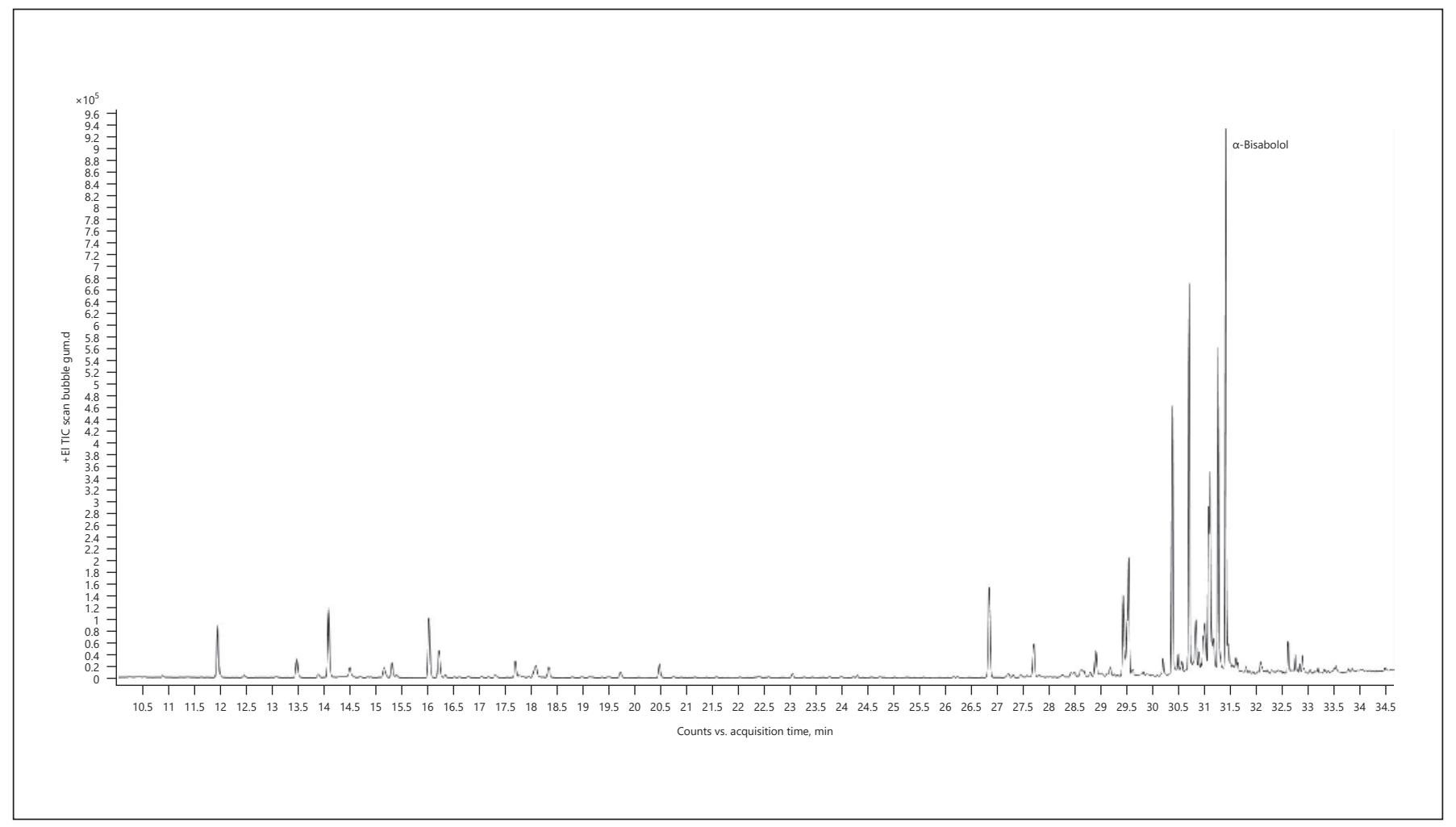

Fig. 16. Gas chromatogram of Bubble Gum cannabis chemotype.

Table 23.

\begin{tabular}{llr}
\hline Normalized \% & Compound & RI \\
\hline $\mathbf{1 0 0 . 0 0}$ & a-Bisabolol & 1,683 \\
62.84 & Bulnesol & 1,666 \\
52.98 & Guaiol & 1,596 \\
19.97 & $\beta$-Myrcene & 991 \\
26.24 & $\beta$-Caryophyllene & 1,418 \\
37.28 & Selina-3,7(11)-diene & 1,542 \\
16.71 & $\gamma$-Eudesmol & 1,631 \\
22.14 & $\beta$-Eudesmol & 1,649 \\
43.24 & $\alpha$-Eudesmol & 1,653 \\
\hline
\end{tabular}

The bold value represents the main compound in the sample. f. Comparison of terpene content in fresh and dry samples:

Chemotype Pandora's Box - fresh (Fig. 17; Table 24); Chemotype Pandora's Box - dry (Fig. 18; Table 25)

g. All identified volatile compounds in 1 chemotype: Chemotype Lemon OG Kush (cannabis inflorescence) (Fig. 19; Table 26);

Chemotype Futura (hemp essential oil) (Fig. 20; Table 27);

Chemotype Black Dream (cannabis essential oil) (Fig. 21; Table 28) 


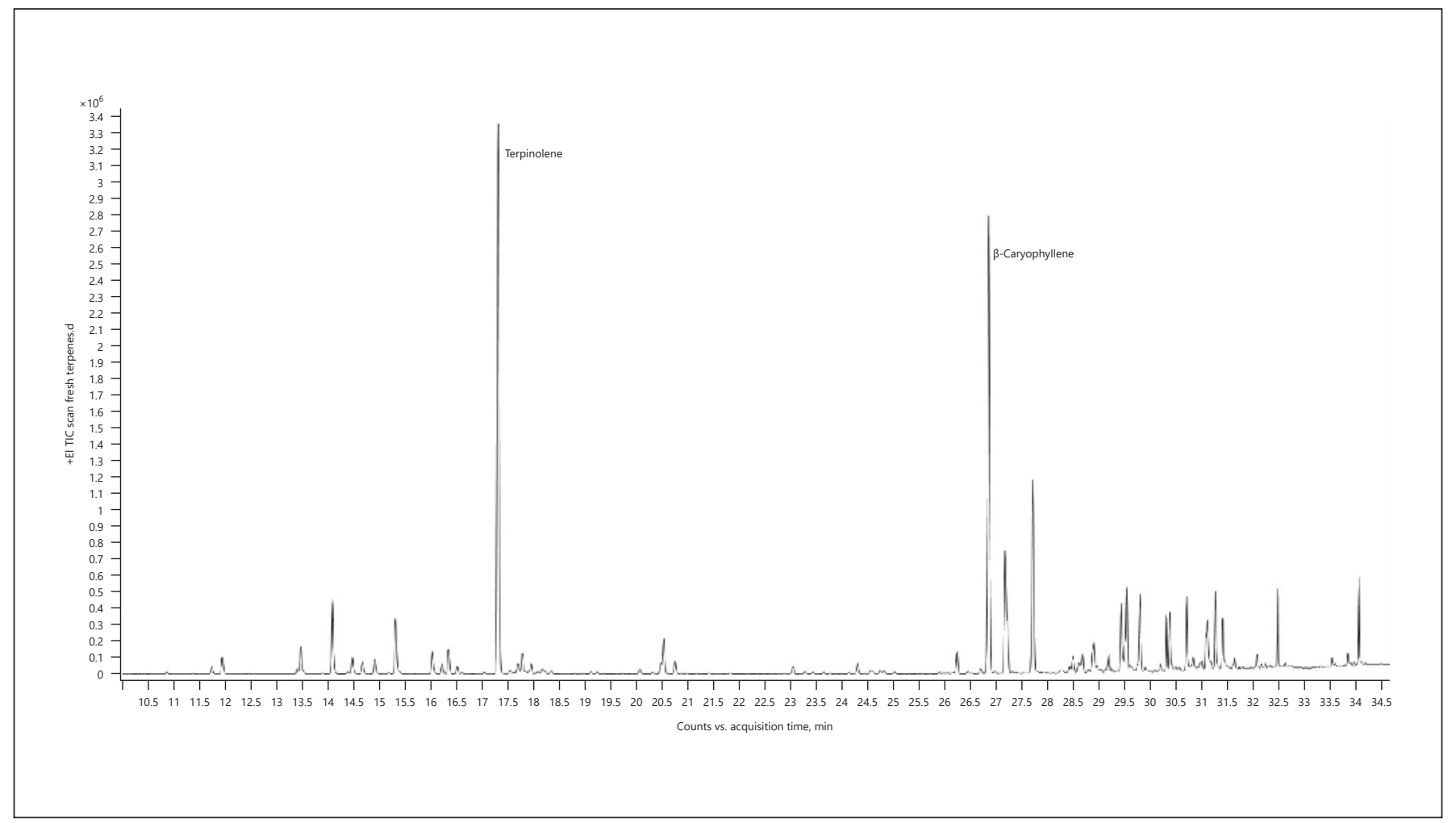

Fig. 17. Gas chromatogram of Pandora's Box cannabis chemotype (fresh sample).

Table 24.

\begin{tabular}{rlr}
\hline Normalized \% & Compound & RI \\
\hline $\mathbf{1 0 0 . 0 0}$ & Terpinolene & 1,088 \\
86.22 & Caryophyllene & 1,419 \\
34.56 & a-Humulene & 1,454 \\
22.67 & $\gamma$-Elemene & 1,434 \\
15.18 & Selina-3,7(11)-diene & 1,542 \\
12.95 & B-Myrcene & 991 \\
11.37 & Germacrene B & 1,556 \\
11.11 & a-Cadinene & 1,538 \\
9.96 & Bulnesol & 1,667 \\
\hline
\end{tabular}

The bold value represents the main compound in the sample. 


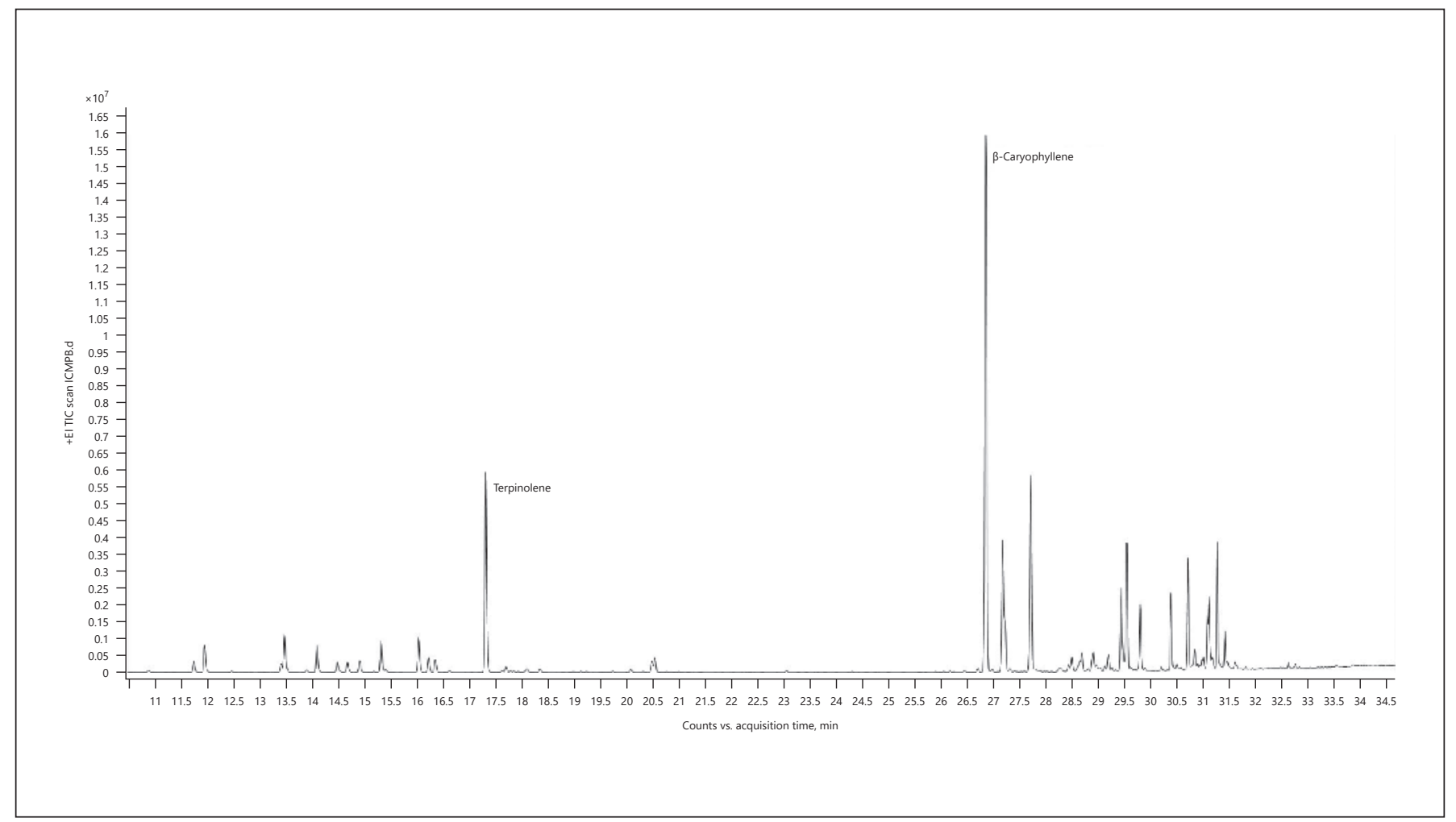

Fig. 18. Gas chromatogram of Pandora's Box cannabis chemotype (dry sample).

Table 25.

\begin{tabular}{cll}
\hline Normalized \% & Compound & RI \\
\hline $\mathbf{1 0 0 . 0 0}$ & $\boldsymbol{\beta}$-Caryophyllene & 1,418 \\
37.19 & Terpinolene & 1,088 \\
35.89 & a-Humulene & 1,454 \\
30.82 & $\gamma$-Elemene & 1,434 \\
20.55 & Selina-3,7(11)-diene & 1,542 \\
15.37 & Bulnesol & 1,667 \\
14.74 & 10-Epi- $\gamma$-eudesmol & 1,619 \\
10.84 & $\alpha$-Eudesmol & 1,653 \\
10.54 & Guaiol & 1,596 \\
10.39 & Germacrene B & 1,556 \\
\hline
\end{tabular}

The bold value represents the main compound in the sample. 


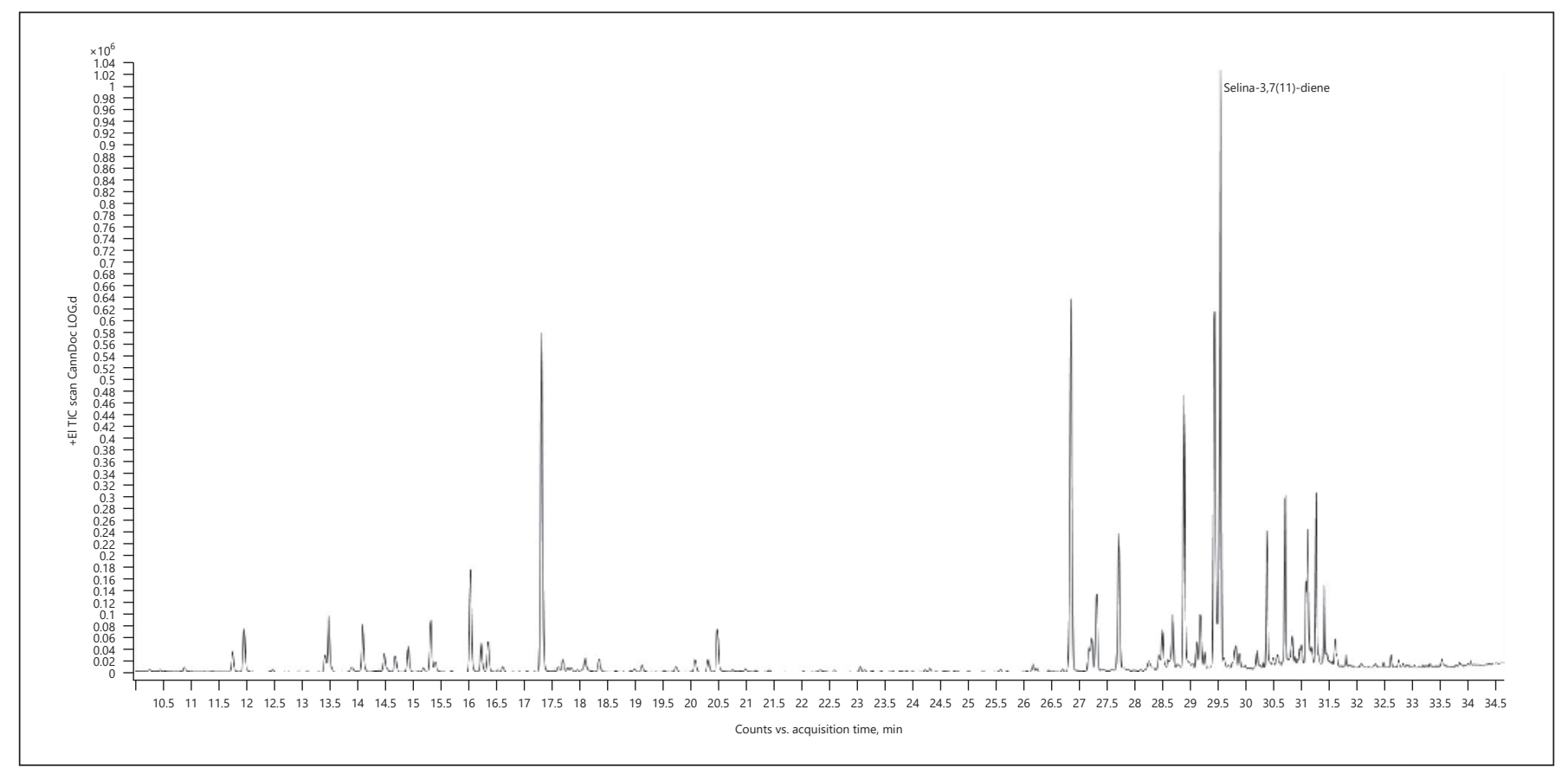

Fig. 19. Gas chromatogram of Lemon OG Kush cannabis chemotype.

Table 26.

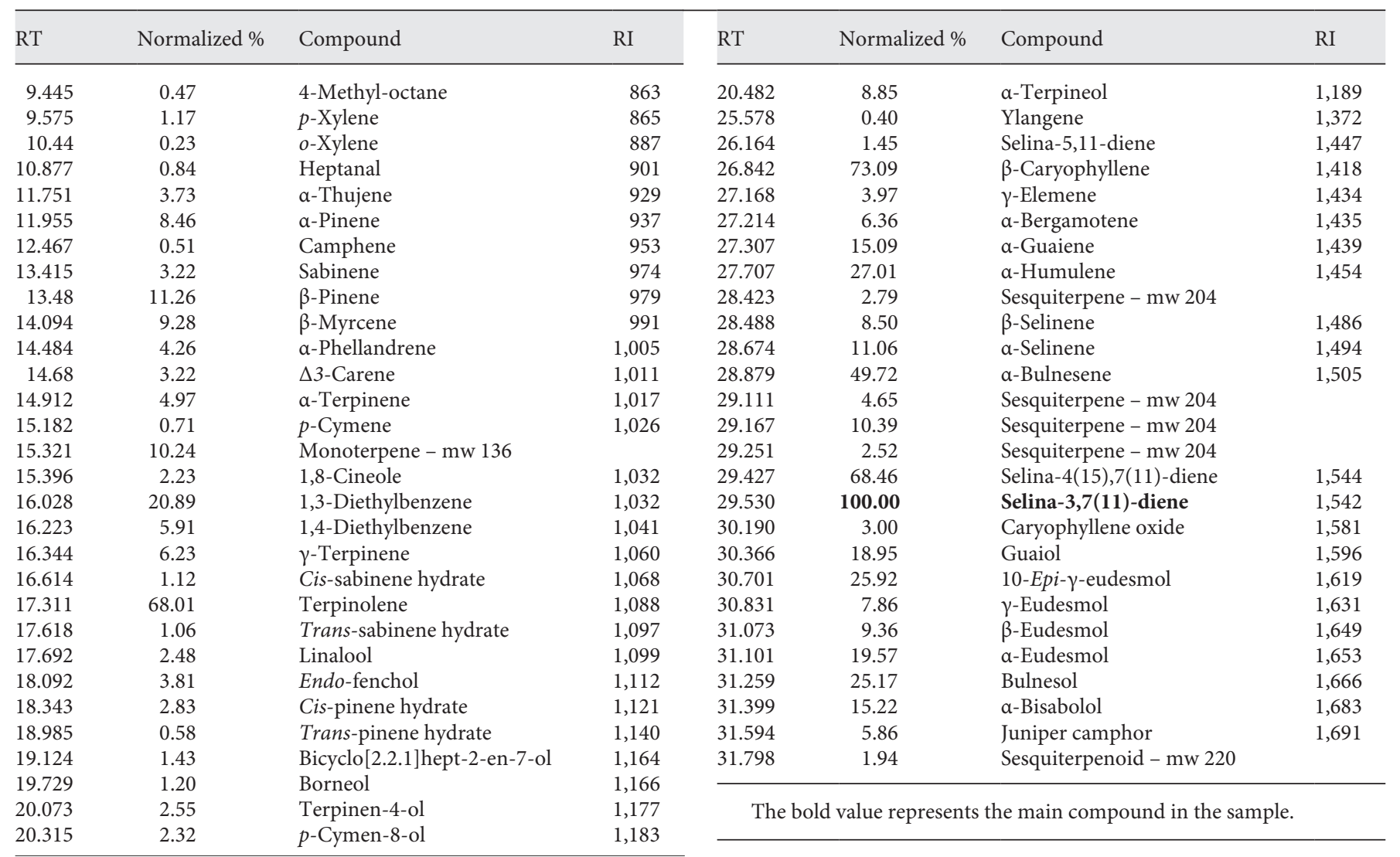

Importance of Terpenes and Terpenoids

Med Cannabis Cannabinoids 2020;3:25-60 DOI: $10.1159 / 000509733$ 


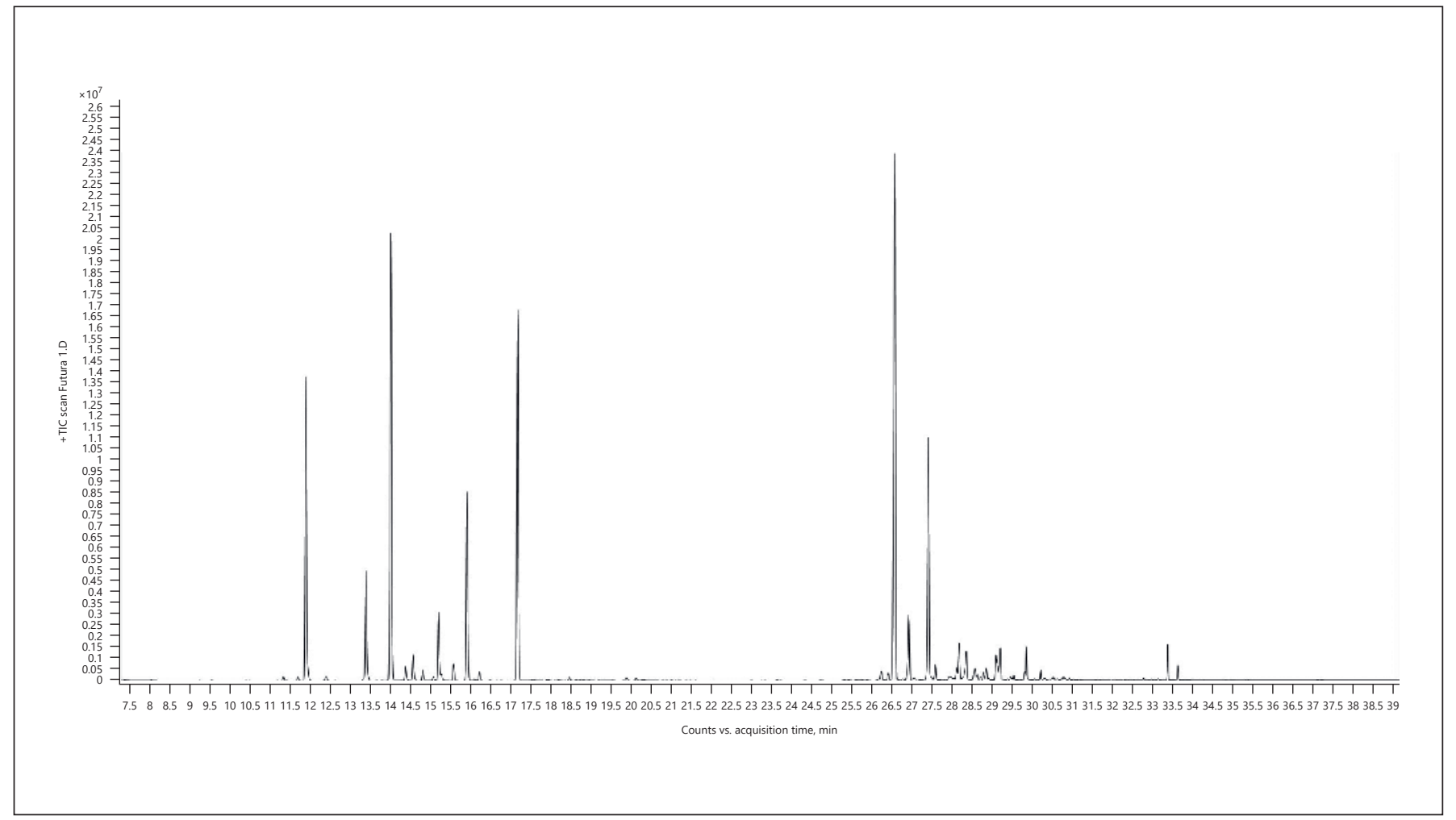

Fig. 20. Gas chromatogram of Futura hemp chemotype. 
Table 27.

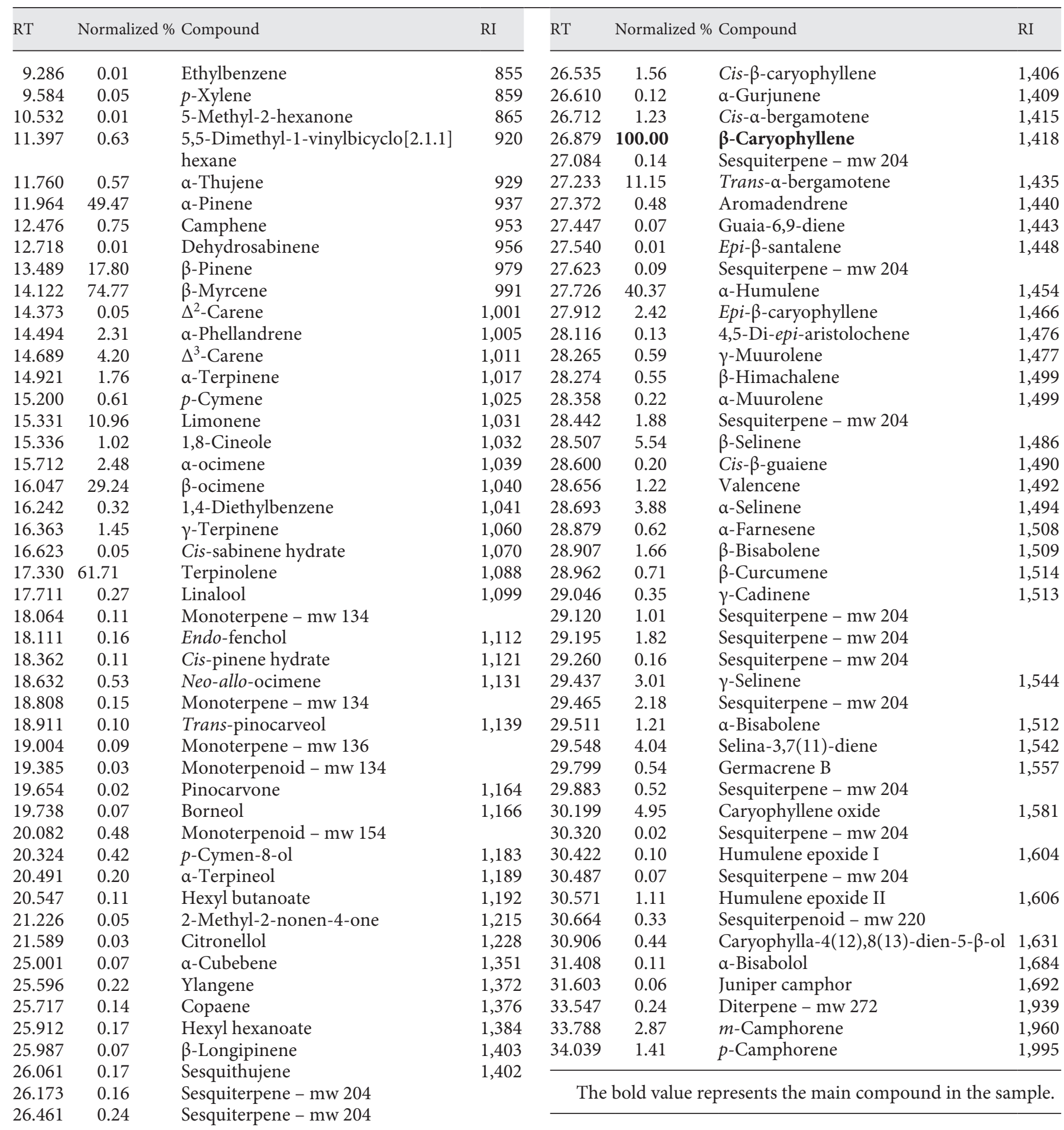




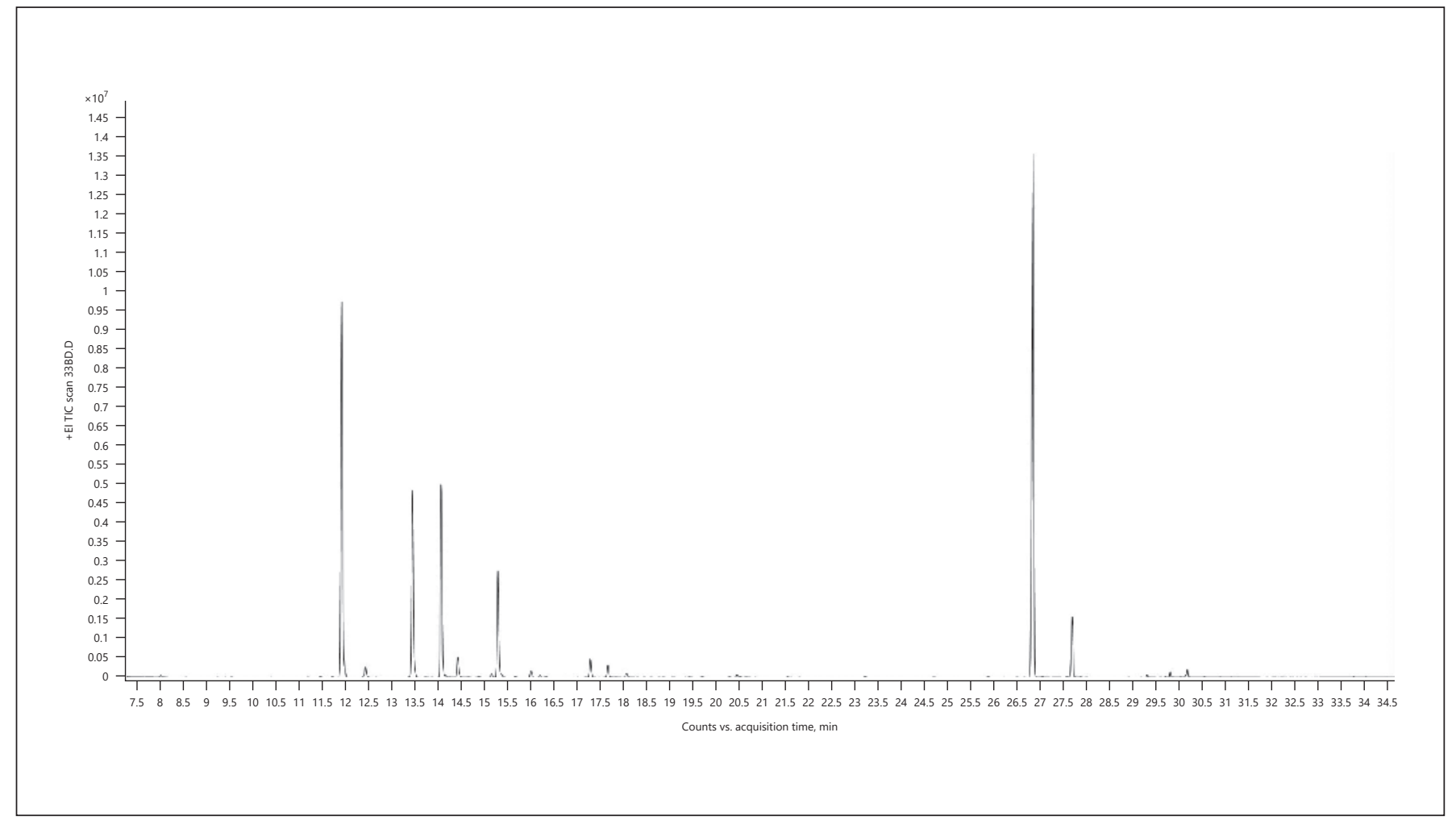

Fig. 21. Gas chromatogram of Black Dream cannabis chemotype. 
Table 28.

\begin{tabular}{|c|c|c|c|}
\hline RT & Normalized \% & Compound & RI \\
\hline 9.268 & 0.02 & Ethylbenzene & 855 \\
\hline 9.565 & 0.06 & p-Xylene & 865 \\
\hline 11.211 & 0.04 & 2,6-Dimethyl-4-octene & 911 \\
\hline 11.481 & 0.18 & Tricyclene & 926 \\
\hline 11.741 & 0.12 & $a$-Thujene & 929 \\
\hline 11.946 & 67.03 & a-Pinene & 937 \\
\hline 12.457 & 2.11 & Camphene & 953 \\
\hline 12.690 & 0.03 & Dehydrosabinene & 956 \\
\hline 13.471 & 34.15 & $\beta$-Pinene & 979 \\
\hline 13.750 & 0.09 & mw 138 & \\
\hline 14.084 & 33.17 & $\beta$-Myrcene & 991 \\
\hline 14.177 & 0.36 & $\mathrm{mw} 138$ & \\
\hline 14.270 & 0.07 & mw 138 & \\
\hline 14.447 & 3.74 & $p$-Mentha-1(7),8-diene & 1,004 \\
\hline 14.680 & 0.10 & $\Delta^{3}$-Carene & 1,011 \\
\hline 14.903 & 0.21 & $\alpha$-Terpinene & 1,017 \\
\hline 15.182 & 0.62 & p-Cymene & 1,026 \\
\hline 15.312 & 18.87 & Limonene & 1,031 \\
\hline 15.386 & 0.78 & 1,8-Cineole & \\
\hline 15.693 & 0.18 & $\alpha$-Ocimene & 1,039 \\
\hline 16.028 & 1.14 & 1,3-Diethylbenzene & 1,032 \\
\hline 16.223 & 0.34 & 1,4-Diethylbenzene & 1,041 \\
\hline 16.344 & 0.24 & $\gamma$-Terpinene & 1,060 \\
\hline 17.218 & 0.05 & Monoterpene - mw 136 & \\
\hline 17.302 & 3.37 & Terpinolene & 1,088 \\
\hline 17.683 & 2.25 & Linalool & 1,098 \\
\hline 18.083 & 0.79 & Endo-fenchol & 1,112 \\
\hline 18.204 & 0.07 & Exo-fenchol & 1,116 \\
\hline 18.613 & 0.04 & Allo-ocimene & 1,129 \\
\hline 18.883 & 0.05 & Trans-pinocarveol & 1,139 \\
\hline 19.096 & 0.07 & Cis-verbenol & 1,142 \\
\hline 19.440 & 0.10 & Isoborneol & 1,157 \\
\hline 19.719 & 0.16 & Borneol & 1,166 \\
\hline 20.305 & 0.12 & p-Cymen-8-ol & 1,183 \\
\hline 20.473 & 0.48 & $a$-Terpineol & 1,189 \\
\hline 20.528 & 0.23 & Hexyl butyrate & 1,192 \\
\hline 20.677 & 0.12 & $\gamma$-Terpineol & \\
\hline 20.872 & 0.02 & mw 152 & \\
\hline 21.561 & 0.13 & Citronellol & 1,228 \\
\hline 21.821 & 0.03 & mw 152 & \\
\hline 23.234 & 0.19 & Bornyl acetate & 1,284 \\
\hline 24.713 & 0.06 & Methyl anthranilate & 1,343 \\
\hline 25.884 & 0.22 & Hexyl hexanoate & 1,384 \\
\hline 26.507 & 0.06 & $\beta$-Cis-caryophyllene & 1,406 \\
\hline 26.851 & 100.00 & $\beta$-Caryophyllene & 1,418 \\
\hline 27.056 & 0.26 & 10,10-Dimethyl-2,6-dimethylenebicyclo[7.2.0]undecane & 1,440 \\
\hline 27.586 & 0.10 & Sesquiterpene/mw 204 & \\
\hline 27.698 & 10.65 & a-Humulene & 1,454 \\
\hline 27.884 & 0.10 & Alloaromadendrene & 1,461 \\
\hline 29.195 & 0.03 & Trans-calamenene & 1,529 \\
\hline 29.316 & 0.38 & Nerolidol & 1,534 \\
\hline 29.808 & 0.80 & Trans-nerolidol & 1,564 \\
\hline 30.041 & 0.07 & Sesquiterpenoid - mw 220 & \\
\hline 30.190 & 1.31 & Caryophyllene oxide & 1,581 \\
\hline 30.552 & 0.09 & Humulene epoxide II & 1,606 \\
\hline 30.887 & 0.05 & 11,11-Dimethyl-4,8-dimethylenebicyclo[7.2.0]undecan-3-ol & \\
\hline 33.770 & 0.11 & $m$-Camphorene & 1,960 \\
\hline 34.030 & 0.04 & $p$-Camphorene & 1,995 \\
\hline
\end{tabular}

The bold value represents the main compound in the sample. 


\section{Discussion}

The purpose of this study was to identify and compare different strains of C. sativa L. with emphasis on terpenes/ terpenoids percentages. We tried to identify the most common, the most abundant, and the most interesting compounds in dry flowering tops and etheric oils of hemp and cannabis.

From the chromatograms and tables, one can see that there are many different chemotypes of $C$. sativa according to their terpenes/terpenoids and that the main volatiles can differ one from the other. This will also influence the industrial, medicinal, and recreational use of this plant. The most important is the content of these volatile compounds in medicine as they will influence the treatment of different diseases.

Comparison of essential oils from hemp and cannabis gave different results. In essential oil of hemp, there were mainly monoterpenes, while in cannabis, the sesquiterpenes/sesquiterpenoids predominate. In any of the 108 chemotypes, the main compounds were $\beta$-caryophyllene, $\beta$-myrcene, $\alpha$-pinene, $\alpha$-humulene, limonene, and $\beta$-pinene.

Between all the 108 chemotypes were the ones where the main terpene/terpenoid was $\beta$-caryophyllene, $\beta$-myrcene, $\alpha$-pinene, limonene, terpinolene, linalool, selina-3,7(11)-diene, $\quad \gamma$-selinene, $\quad 10$-epi- $\gamma$-eudesmol, $\beta$-eudesmol, $\alpha$-eudesmol, bulnesol, or $\alpha$-bisabolol. In plant material (inflorescence) of cannabis (54 chemotypes), the main compounds were $\beta$-caryophyllene, $\beta$-myrcene, guaiol, 10-epi- $\gamma$-eudesmol, selina-3,7(11)-diene, and $\alpha$-humulene.

When we evaluated hemp and cannabis inflorescence and essential oil samples for 10 the main terpenes in any from 108 analyzed chemotypes, between them were 58 different terpenes/terpenoids. From these 44 different terpenes/terpenoids were found in 54 chemotypes of cannabis inflorescence samples, 27 different ones in 46 chemotypes of cannabis essential oils, and 17 different ones were found in 8 chemotypes of hemp essential oils. Sometimes we can see that the main terpene/terpenoid does not reach $20 \%$ of total ones in the analyzed sample: Kilimanjaro a-pinene (17.45\%) (Fig. 4); Durban Poison - $\beta$-myrcene (16.75\%) (Fig. 5); Edom - $\beta$-caryophyllene (15.74\%) (Fig. 9); Lemon OG Kush - selina-3,7(11)-diene (10.03\%) (Fig. 10); Fuji - $\gamma$-selinene (10.85\%) (Fig. 11); Lemon Deluxe - 10-epi- $\gamma$-eudesmol (10.37\%) (Fig. 12); Alaska $\beta$-eudesmol (8.96\%) (Fig. 13); El Na- $\alpha$-eudesmol (9.66\%) (Fig. 14); Berry Deluxe - bulnesol (14.72\%) (Fig. 15); and Bubble Gum - a-bisabolol (16.03\%) (Fig. 16).
Table 29. Inflorescences of cannabis chemotypes (the main terpene in the sample is above $20 \%$ of total)

\begin{tabular}{lll}
\hline Chemotype & $\begin{array}{l}\text { \% of total terpenes/ } \\
\text { terpenoids }\end{array}$ & Terpene/terpenoid \\
\hline Doblin & 33.37 & $\beta$-Caryophyllene \\
\hline Jericho & 30.21 & $\alpha$-Pinene \\
\hline White widow & 27.51 & $\alpha$-Pinene \\
\hline Pandora's box dry & 25.32 & $\beta$-Caryophyllene \\
\hline Tel Aviv & 24.99 & $\alpha$-Pinene \\
\hline Pandora's box fresh & 24.55 & Terpinolene \\
\cline { 2 - 3 } & 21.16 & $\beta$-Caryophyllene \\
\hline DOV & 22.45 & $\beta$-Myrcene \\
\hline Maui Waui & 22.09 & Selina-3,7(11)-diene \\
\hline Kira Kush & 21.61 & Selina-3,7(11)-diene \\
\hline Jack Herer & 21.39 & Terpinolene \\
\hline Kush & 20.66 & $\alpha$-Pinene \\
\hline
\end{tabular}

Table 30. Hemp essential oils (the main terpene in the sample is above $20 \%$ of total)

\begin{tabular}{lll}
\hline Chemotype & $\begin{array}{l}\text { \% of total terpenes/ } \\
\text { terpenoids }\end{array}$ & Terpene/terpenoid \\
\hline Tisza & 27.60 & $\beta$-Caryophyllene \\
Fedora & 26.19 & $\beta$-Caryophyllene \\
Ferimon & 23.63 & $\beta$-Caryophyllene \\
Futura & 21.47 & $\beta$-Caryophyllene \\
Tiborszallasi & 21.27 & $\beta$-Myrcene \\
Felina & 21.14 & $\beta$-Myrcene \\
\hline
\end{tabular}

It is also worth to discuss degree of terpenes/terpenoids diversity, since it is very different between various chemotypes. Unfortunately, we did not have access to the hemp inflorescence, so we just present only inflorescences of different cannabis chemotypes (Table 29). When we compare hemp essential oil (Table 30) and cannabis essential oil (Table 31), it became clear that almost in all cannabis essential oil samples, the concentration of the main terpene/terpenoid is higher than that in hemp ones. Moreover, in 8 cases, concentration of the main terpene/ terpenoid in cannabis essential oil is higher than $50 \%$ of total amount of terpenes/terpenoids. It is interesting that the main terpenes/terpenoids above $20 \%$ in the analyzed 
Table 31. Cannabis essential oils (the main terpene/s in the sample is above $20 \%$ of total)

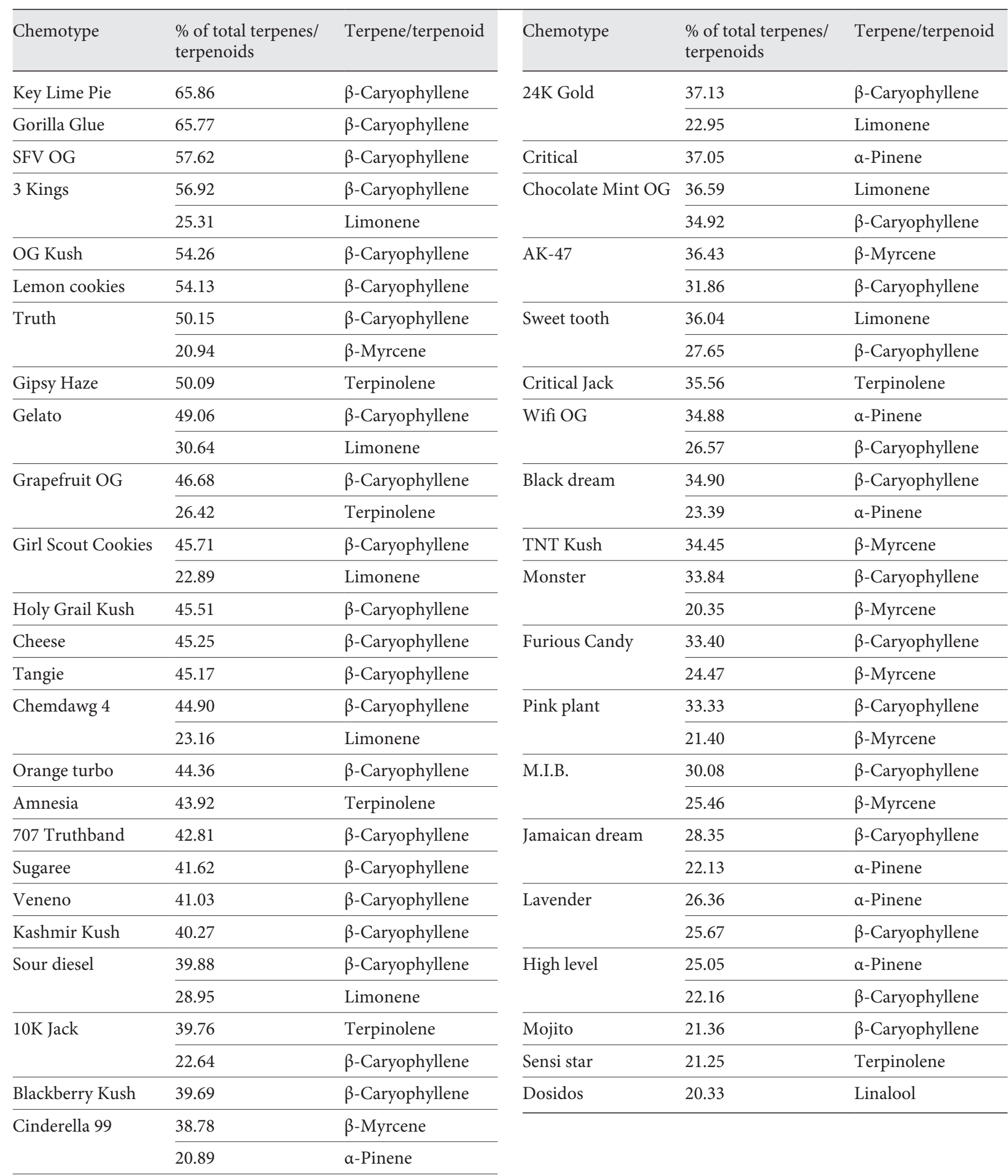




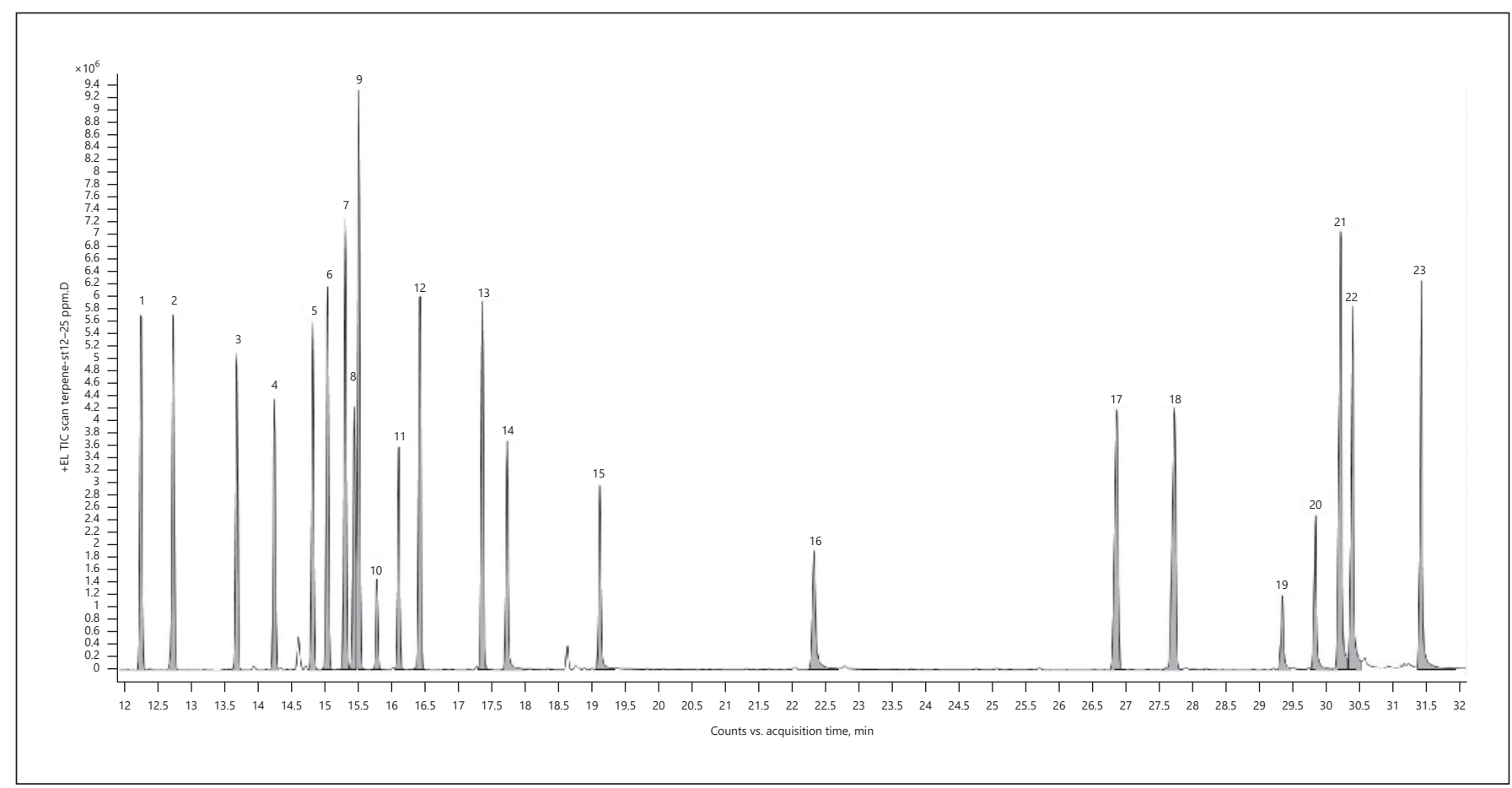

Fig. 22. GC/MS chromatogram of 23 terpenes/terpenoids standards (RESTEK Catalog. No. 34095 and 34096). Key: 1, $\alpha$-pinene; 2 , camphene; $3,(-)$ - $\beta$-pinene; $4, \beta$-myrcene; $5, \Delta 3$-carene; 6 , $\alpha$-terpinene; 7, $p$-cymene; $8, d$-limonene; $9,1,8$-cineole; (10, 31\% $\alpha$-ocimene $+11,69 \% \beta$-ocimene); $12, \gamma$-terpinene; 13 , terpinolene; 14, linalool; 15 , (-)-isopulegol; 16 , geraniol; $17, \beta$-caryophyllene; 18, $\alpha$-humulene; (19, 39\% cis-nerolidol $+20,61 \%$ trans-nerolidol); 21, (-)-caryophyllene oxide; 22, (-)-guaiol; 23, (-)-a-bisabolol. samples were only $\beta$-caryophyllene (39x), $\alpha$-pinene $(8 \times)$, $\beta$-myrcene $(8 \times)$, terpinolene $(8 \times)$, limonene $(8 \times)$, selina3,7(11)-diene $(2 \times)$, and linalool $(1 \times)$.

$\beta$-Caryophyllene and $\beta$-myrcene are without any doubt the most common (and often the main ones) terpenes in C. sativa L. plant. Because of their unique properties, these are probably the terpenes that play an important role in medicinal properties of hemp and cannabis.

We would like to inform readers also about the monoterpenes/monoterpenoids-sesquiterpene/sesquiterpenoids ratio in the analyzed samples. Our results show that this ratio does not depend if the sample is hemp, cannabis, or essential oils from them. The content of monoterpenes/monoterpenoids prevailed in most samples, sometimes several times more. Very rarely, the content of sesquiterpenes/sesquiterpenoids was higher, but only slightly more. There is a difference between fresh and dry plant monoterpene content, and between etheric oil and organic solvent extract (it also depends on the type of solvent). Different work-up of different solvent extracts can lead to the loss of monoterpenes. Essential oil and organ- ic solvent extract constituents can be different before and after drying process, and before and after storage. A considerably lower content of monoterpenes can be found in the solvent extract compared with the essential oil from steam distillation. Steam distillation also promotes dehydration of labile alcohols.

The most important is which type of information we need. From biogenetic point of view, the best is headspace gas chromatography of the fresh plant material immediately after its harvest. From pharmacological point of view, the best is to analyze the preparation which is used for medicinal purposes. It is without any doubt that in the course from the living material in the plant to the product we are analyzing, there is a loss of volatile substances, especially monoterpenes.

Many terpenes/terpenoids have a therapeutic power in their mixtures with or without phytocannabinoids, and can be very useful and sometime powerful in treatment of diseases. In the Introduction, we have mentioned several terpenes and their potential medicinal properties. We found more therapeutically interesting structures upon doing our analyses. For instance, 10-epi- $\gamma$-eudesmol is 
Table 32. The relative content of terpenes (\%) in the sample analyzed by GC/MS

\begin{tabular}{|c|c|c|c|}
\hline Peak & Compound & $\%$ & Normalized, \% \\
\hline 1 & a-Pinene & 4.77 & 61.98 \\
\hline 2 & Camphene & 4.77 & 61.96 \\
\hline 3 & $\beta$-Pinene & 4.28 & 55.58 \\
\hline 4 & Myrcene & 3.39 & 44.04 \\
\hline 5 & $\Delta^{3}$-Carene & 4.61 & 59.88 \\
\hline 6 & a-Terpinene & 5.09 & 66.16 \\
\hline 7 & p-Cymene & 6.15 & 79.84 \\
\hline 8 & Limonene & 3.81 & 49.50 \\
\hline 9 & 1,8-Cineole & 7.70 & 100.00 \\
\hline 10 & a-Ocimene (31\%) & 1.12 & 14.51 \\
\hline 11 & $\beta$-Ocimene $(69 \%)$ & 2.92 & 37.96 \\
\hline 12 & $\gamma$-Terpinene & 5.05 & 65.55 \\
\hline 13 & Terpinolene & 4.97 & 64.54 \\
\hline 14 & Linalool & 3.19 & 41.46 \\
\hline 15 & Isopulegol & 2.61 & 33.89 \\
\hline 16 & Geraniol & 2.53 & 32.93 \\
\hline 17 & $\beta$-Caryophyllene & 5.59 & 72.56 \\
\hline 18 & a-Humulene & 5.72 & 74.29 \\
\hline 19 & Cis-nerolidol (38\%) & 1.20 & 15.59 \\
\hline 20 & Trans-nerolidol (61\%) & 2.36 & 30.67 \\
\hline 21 & Caryophyllene oxide & 7.59 & 98.58 \\
\hline 22 & Guaiol & 5.50 & 71.51 \\
\hline 23 & a-Bisabolol & 5.09 & 66.18 \\
\hline
\end{tabular}

highly effective against melanoma and column carcinoma cells proliferation [108]; $\beta$-eudesmol is antihepatotoxic, antiangiogenic, and antitumor [109-111]; a-eudesmol induces apoptosis [112]; bulnesol possesses antitussive and expectorant activity [113]; a-bisabolol induces apoptosis of malignant tumor cells, cytotoxicity, and antigenotoxicity [114-118]; guaiol is an anti-inflammatory, antimicrobial, and analgesic terpenoid [70, 71]; and $\alpha$-humulene acts as an appetite suppressant, antibacterial, and antitumor agent and is an effective anti-inflammatory and analgesic sesquiterpene [73, 119]. Monoterpenoid constituents have antioxidant, anti-inflammatory, and estrogenic effects, and these activities are also relevant to current Alzheimer's disease therapy. Several monoterpenoid alcohols demonstrated good anti-Parkinson's disease activity. Many monoterpenoids demonstrated promising neuroprotective activity mediated by various systems [120]. There are some other important terpenes such as selina-3,7(11)-diene or $\gamma$-selinene which have never been tested for their pharmacological properties.
We must realize that if we evaluate the relative content of terpenes (\%) in the sample, it has nothing to do with their quantitative content in the plant. It is clear from Figure 22 and Table 32 that when we analyze a mixture of standards, where each one has the same concentration (25 ppm in hexane), the area of some peaks is up to 3 times smaller than the area of the largest peak. This is very good for comparing samples and the ratio of the content terpenes/terpenoids, but not for quantifying them.

Today, we know that in the brain concentrations of anandamide (AEA) are in picomoles and 2-arachidonoylglycerol (2-AG) in nanomoles (what is ng AEA/g brain or $\mu$ g 2-AG/g brain) [121]. Terpenes/terpenoids are in dried cannabis flower material (which is used for medicinal purposes) usually at concentrations $\mathrm{mg} / \mathrm{g}$ [122]. As mentioned in Casano et al. [123], "the relative content of terpenoids is strongly inherited while total yield per weight of tissue is more subjected to environmental factors." Relative content (\%) of terpenes/terpenoids is more often used for chemosystematic studies (and it was used also in this publication). What can we deduce from the previous few sentences? Receptors need their ligands in very low concentrations, so it is quite possible that these terpenes/terpenoids are important in the treatment, which are in the plant in very low concentrations and which we are not considering today.

The healing power of cannabis most likely resides in terpenes/terpenoids and phytocannabinoids, of which particular compounds, their amount, and the ratio to each other play the most important role in the treatment of particular diseases. It should be emphasized here that flavonoids, flavonoid glycosides, polyphenol, and the other biodynamic compounds will play this game too.

\section{Acknowledgements}

I would like to express my special appreciation and thanks to Mr. Boris Valte from Slovenia and to Mr. Leopold Svatý from the Czech Republic for hemp essential oils.

\section{Statement of Ethics}

The authors have no ethical conflicts to disclose.

\section{Conflict of Interest Statement}

The authors have no conflicts of interest to declare. 


\section{Funding Sources}

There were no external funding sources to the study in the preparation of data or the manuscript.

\section{Author Contributions}

Both authors contributed equally to the manuscript.

\section{References}

1 Strainprint Technologies Inc., Toronto, Canada.

2 Hanuš LO. Pharmacological and therapeutic secrets of plant and brain (endo)cannabinoids. Med Res Rev. 2009;29(2):213-71.

3 Krejčí Z. Antibioticky princip Cannabis indica. (The antibiotic effect of Cannabis indica.) Brno, Czechoslovakia: Masaryk University; 1950. p. 106.

4 Krejčí $Z$. The antibacterial effect of Cannabis indica. Lék listy. 1952;7:500.

5 Konopí jako lék. (Cannabis as a medicine.). Acta Univ Palacki Olomuc. 1955;6:27-114.

6 Krejčí Z, Šantavý F. Isolace dalších látek z listí indického konopí Cannabis sativa L. (Isolation of other substances from the leaves of the Indian hemp (Cannabis sativa L., varietas indica). Acta Univ Palacki Olomuc. 1955;6:59_ 66.

7 Krejčí Z, Horák M, Šantavý F. Konstituce kyseliny kanabidiolové a kyseliny b.t. $133^{\circ} \mathrm{C}$ isolovaných Z Cannabis sativa L. (Constitution of the cannabidiolic acid and of an acid of the M.P. 133, isolated from Cannabis sativa L.) Acta Univ Palacki Olomuc. 1958;16:9-17.

8 Hanuš L, Krejčí Z, Hruban L. Isolation of cannabidiolic acid from Turkish variety of cannabis cultivated for fibre. Acta Univ Olomuc Fac Med. 1975;74:167-72.

9 Kabelík J, Krejčí Z, Šantavý F. Cannabis as a medicament. Bull Narc. 1960;12:5-23.

10 Krejčí Z. Antibakterielní látky v prevenci i terapii infekcí. (The antibacterial substances from cannabis in the treatment and prevention of infections.) Olomouc, Czechoslovakia: Palacký University; 1961. p. 259.

11 Jacob A, Todd AR. Cannabis indica. Part II. Isolation of cannabidiol from Egyptian hashish. Observations on the structure of cannabinol. J Chem Soc. 1940;649-53.

12 Ghosh R, Todd AR, Wilkinson S. Cannabis indica. Part V. The synthesis of cannabinol. J Chem Soc. 1940;1393-6.

13 Adams R, Baker BR, Wearn RB. Structure of cannabinol. III. Synthesis of cannabinol, 1-Hydroxy-3-n-amyl-6,6,9-trimethyl6-dibenzopyran. J Amer Chem Soc. 1940;62: 2204-7.

14 Hanuš L, Tesařík K, Krejčí Z. Capillary gas chromatography of natural substances from Cannabis sativa L. I. Cannabinol and cannabinolic acid: artefacts. Acta Univ Palacki Olomuc Fac Med. 1985;108:29-38.

15 Adams R, Wolff H, Cain CK, Clark JH. Structure of cannabidiol. V. Position of the alicyclic double bonds. J Amer Chem Soc. 1940;62: 2215-9.
16 Mechoulam R, Shvo Y. Hashish-I. Tetrahedron. 1963;19(12):2073-8.

17 Adams R, Pease DC, Cain CK, Baker BR, Clark JH, Wolff H, et al. Conversion of cannabidiol to a product with marihuana activity. A type reaction for synthesis of analogous substances. Conversion of cannabidiol to cannabinol. J Amer Chem Soc. 1940;62:2245-6.

18 Gaoni Y, Mechoulam R. Isolation, structure, and partial synthesis of an active constituent of hashish. J Am Chem Soc. 1964;86(8): 1646-7.

19 Šantavý F. Notes on the structure of cannabidiol compounds. Acta Univ Palacki Olomuc Fac Med. 1964;35:5-9.

20 Hanuš LO, Meyer SM, Muñoz E, TaglialatelaScafati O, Appendino G. Phytocannabinoids: a unified critical inventory. Nat Prod Rep. 2016;33:1357-92.

21 Berman P, Futoran K, Lewitus GM, Mukha D Benami M, Shlomi T, et al. A new ESI-LC/MS approach for comprehensive metabolic profiling of phytocannabinoids in Cannabis. Sci Rep. 2018;8(1):14280.

22 Citti C, Linciano P, Russo F, Luongo L, Iannotta $\mathrm{M}$, Maione $\mathrm{S}$, et al. A novel phytocannabinoid isolated from Cannabis sativa $\mathrm{L}$. with an in vivo cannabimimetic activity higher than $\Delta 9$-tetrahydrocannabinol: $\Delta 9$ tetrahydrocannabiphorol. Sci Rep. 2019;9(1): 20335.

23 Basas-Jaumandreu J, de las Heras FXC. GCMS metabolite profile and identification of unusual homologous cannabinoids in high potency Cannabis sativa. Planta Med. 2020; 86(5):338-347.

24 Devane WA, Dysarz FA, Johnson MR, Melvin LS, Howlett AC. Determination and characterization of a cannabinoid receptor in rat brain. Mol Pharmacol. 1988;34(5):605-13.

25 Matsuda LA, Lolait SJ, Brownstein MJ, Young AC, Bonner TI. Structure of a cannabinoid receptor and functional expression of the cloned cDNA. Nature. 1990;346(6284): 561-4.

26 Devane WA, Hanuš L, Breuer A, Pertwee RG, Stevenson LA, Griffin G, et al. Isolation and structure of a brain constituent that binds to the cannabinoid receptor. Science. 1992; 258(5090):1946-9.

27 Hanuš LO. Discovery and isolation of anandamide and other endocannabinoids, Chapter 12, p. 1828-41. In: Lambert DM, editor. Cannabinoids in nature and medicine. $\mathrm{Zu}$ rich: Verlag Helvetica Chimica Acta, WileyVCH; 2009. p. 416.
28 Hanuš LO. Discovery and isolation of anandamide and other endocannabinoids. Chem Biodivers. 2007;4(8):1828-41.

29 Hanuš L, Gopher A, Almog S, Mechoulam R. Two new unsaturated fatty acid ethanolamides in brain that bind to the cannabinoid receptor. J Med Chem. 1993;36(20):3032-4.

30 Mechoulam R, Ben-Shabat S, Hanuš L, Ligumsky M, Kaminski NE, Schatz AR, et al. Identification of an endogenous 2-monoglyceride, present in canine gut, that binds to cannabinoid receptors. Biochem Pharmacol. 1995;50(1):83-90.

31 Hanuš L, Abu-Lafi S, Fride E, Breuer A, Vogel Z, Shalev DE. 2-Arachidonyl glyceryl ether, a novel endogenous agonist of the cannabinoid CB1 receptor. Proc Natl Acad Sci U S A. 2001; 98(7):3662-5.

32 Bonn-Miller M, Pollack MO Jr, Casarett D, Dart R, ElSohly M, Good L, et al. Priority considerations for medicinal cannabis-related research. Cannabis Cannabinoid Res. 2019; 4(3):139-57.

33 Bohlig JF. Cannabis sativa und Urtica dioica, chemisch analysirt. Jahrbuch für practische Pharmacie und verwandte Fächer, herausgegeben von der pharmaceutischen Gesellschaft der Pfalz, Verlag von J. J. Tascher, Kaiserslautern, 1840. p. 1-58.

34 Bolas T, Francis EEH. XXXV.-On the products of the action of nitric acid on the resinous extract of Indian hemp. J Chem Soc. 1869;22: 417-9.

35 Valente L. Sull'essenza di canapa. [Essential oil from hemp.]. Gazz Chim Ital. 1880;10: 479-81.

36 Valente L. Sull'idrocarburo estratto della canapa. [On the hydrocarbon extract of Indian hemp.]. Gazz Chim Ital. 1881;11:196-8.

37 Valente L. Studi sull'essenza di canapa. Atti della Reale Academia dei Lincei. 1881;5: 126-8.

38 Vignolo G. Sull'essenza di Cannabis indica. Atti della Reale Accademia dei Lincei. 1894; 5(3):404-7.

39 Vignolo G. Sull'essenza di Cannabis indica. (Essence of Cannabis indica.). Gazz Chim Ital. 1895;25(i):110-4.

40 Wood TB, Spivey WTN, Easterfield TH. XL.Charas. The resin of Indian hemp. J Chem Soc Trans. 1896;69:539-46.

41 Simonsen JL, Todd AR. Cannabis indica. Part $\mathrm{X}$. The essential oil from Egyptian hashish. J Chem Soc. 1942;1942(1):188-91.

42 Ružička L. The isoprene rule and the biogenesis of terpenic compounds. Experientia. 1953;50(4):395-405. 
43 Kekulé A. Lehrbuch der organischen Chemie. Erlangen, Germany: Ferdinand Enke; 1866. Vol. 2; p. 464

44 Ben-Shabat S, Fride E, Sheskin T, Tamiri T, Rhee MH, Vogel Z, et al. An entourage effect: inactive endogenous fatty acid glycerol esters enhance 2-arachidonoyl-glycerol cannabinoid activity. Eur J Pharmacol. 1998;353(1):23-31.

45 Russo EB. The case for the entourage effect and conventional breeding of clinical cannabis: no "strain," no gain. Front Plant Sci. 2018; 9:1969.

46 Blasco-Benito S, Seijo-Vila M, Caro-Villalobos M, Tundidor I, Andradas C, GarcíaTaboada E, et al. Appraising the "entourage effect": antitumor action of a pure cannabinoid versus a botanical drug preparation in preclinical models of breast cancer. Biochem Pharmacol. 2018 Nov;157:285-93.

47 Krejčí Z, Hanuš L, Yoshida T, Braenden OJ. The effect of climatic and ecologic conditions upon the formation and the amount of cannabinoid substances in the cannabis of various provenance. Acta Univ Olomuc Fac Med. 1975;74:147-60.

48 Hanuš L, Krejčí Z. Dynamics of changes in the content of cannabinoid substances during the vegetation period of Cannabis sativa L. Acta Univ Palacki Olomuc Fac Med. 1986;114:1129.

49 Hanuš L, Šubová D. The amount of main cannabinoid substances in hemp, cultivated for industrial fibre production and their changes in the course of one vegetation period. Acta Univ Palacki Olomuc Fac Med. 1989;122:11-23.

50 Hanuš L, Dostálová M. The effect of soil fertilization on the formation and the amount of cannabinoid substances in Cannabis sativa L. in the course of one vegetation period. Acta Univ Palacki Olomuc Fac Med. 1994;138:11-5.

51 Aizpurua-Olaizola O, Soydaner U, Öztürk E, Schibano D, Simsir Y, Navarro P, et al. Evolution of the cannabinoid and terpene content during the growth of Cannabis sativa plants from different chemotypes. J Nat Prod. 2016 79(2):324-31.

52 Koltai H, Namdar D. Cannabis phytomolecule 'entourage': from domestication to medical use. Trends Plant Sci. 2020.

53 Zaklin R. Terpene therapy. Abstracts of $\mathrm{Pa}$ pers, 256th ACS National Meeting \& Exposition, Boston, MA, August 19-23, 2018 (2018), CHAS-50.

54 Gulluni N, Re T, Loiacono I, Lanzo G, Gori L, Macchi C, et al. Cannabis essential oil: a preliminary study for the evaluation of the brain effects. Evid Based Complement Alternat Med. 2018;2018:1709182.

55 Jansen C, Shimoda LMN, Kawakami JK, Ang L, Bacani AJ, Baker JD, et al. Myrcene and terpene regulation of TRPV1. Channels. 2019; 13(1):344-66

56 Kozioł A, Stryjewska A, Librowski T, Sałat K, Gaweł M, Moniczewski A, et al. An overview of the pharmacological properties and potential applications of natural monoterpenes. Mini Rev Med Chem. 2014;14(14):1156-68.
57 Gertsch J, Leonti M, Raduner S, Racz I, JianZhong C, Xiang-Qun X, et al. Beta-caryophyllene is a dietary cannabinoid. Proc Nat Acad Sci U S A. 2008;105(26):9099-104.

58 Sharma C, Al Kaabi JM, Nurulain SM, Goyal SN, Kamal MA, Ojha S. Polypharmacological properties and therapeutic potential of $\beta$-caryophyllene: a dietary phytocannabinoid of pharmaceutical promise. Curr Pharm Des. 2016;22(21):3237-64.

59 Francomano F, Caruso A, Barbarossa A, Fazio A, La Torre C, Ceramella J, et al. $\beta$-Caryophyllene: a sesquiterpene with countless biological properties. Appl Sci. 2019; 9(24):5420

60 Astani A, Reichling J, Schnitzler P. Screening for antiviral activities of isolated compounds from essential oils. Evid Based Complement Alternat Med. 2011;2011:253643.

61 Salehi B, Upadhyay S, Orhan IE, Jugran AK, Jayaweera SLD, Dias DA, et al. Therapeutic potential of $\alpha$ - and $\beta$-pinene: a miracle gift of nature. Biomolecules. 2019;9:738.

62 Erasto P, Viljoen AM. Limonene: a review: biosynthetic, ecological and pharmacological relevance. Nat Prod Commun. 2008;3:1193202.

63 Sun J. D-limonene: safety and clinical applications. Alt Med Rev. 2007;12:259-64.

64 Kamatou GPP, Viljoen AM. Linalool: a review of a biologically active compound of commercial importance. Nat Prod Commun. 2008;3:1183-92.

65 Khaleel C, Tabanca N, Buchbauer G. $\alpha$-Terpineol, a natural monoterpene: a review of its biological properties. Open Chem. 2018; 16(1):349-61.

66 Fidyt K, Fiedorowicz A, Strzadała L, Szumny A. $\beta$-Caryophyllene and $\beta$-caryophyllene oxide-natural compounds of anticancer and analgesic properties. Cancer Med. 2016;5(10): 3007-17.

67 Zhang JH, Sun HL, Chen SY, Zeng L, Wang TT. Anti-fungal activity, mechanism studies on $\alpha$-phellandrene and nonanal against Penicillium cyclopium. Bot Stud. 2017;58(1):13.

68 Thakre AD, Mulange SV, Kodgire SS, Zore GB, Karuppayil SM. Effects of cinnamaldehyde, ocimene, camphene, curcumin and farnesene on Candida albicans. AiM. 2016; 6(9):627-43.

69 Vallianou I, Hadzopoulou-Cladaras M. Camphene, a plant derived monoterpene, exerts its hypolipidemic action by affecting SREBP-1 and MTP expression. PLoS One. 2016;11(1): e0147117.

70 Yang Q, Wu J, Luo Y, Huang N, Zhen N, Zhou $\mathrm{Y}$, et al. (-)-Guaiol regulates RAD51 stability via autophagy to induce cell apoptosis in nonsmall cell lung cancer. Oncotarget. 2016; 7(38):62585-97.

71 Yang X, Zhu J, Wu J, Huang N, Cui Z, Luo Y, et al. (-)-Guaiol regulates autophagic cell death depending on mTOR signaling in NSCLC. Cancer Biol Ther. 2018;19(8):706-14.
72 Jang H-I, Ki-Jong R, Eom Y-B. Antibacterial and antibiofilm effects of $\alpha$-humulene against Bacteroides fragilis. Can J Microbiol. 2020;66: 389-399.

73 Rogerio AP, Andrade EL, Leite DF, Figueiredo CP, Calixto JB. Preventive and therapeutic anti-inflammatory properties of the sesquiterpene alpha-humulene in experimental airways allergic inflammation. Br J Pharmacol. 2009;158(4):1074-87.

74 Legault J, Dahl W, Debiton E, Pichette A, Madelmont JC. Antitumor activity of balsam fir oil: production of reactive oxygen species induced by alpha-humulene as possible mechanism of action. Planta Med. 2003; 69(5):402-7.

75 Guimarães AG, Quintans JS, Quintans LJ Jr. Monoterpenes with analgesic activity: a systematic review. Phytother Res. 2013;27(1):115.

76 Ramalho TR, Pacheco de Oliveira MT, Lima AL, Bezerra-Santos CR, Piuvezam MR. Erratum for: gamma-terpinene modulates acute inflammatory response in mice. Planta Med. 2015;81(14):E3-54.

77 Yao YQ, Ding X, Jia YC, Huang CX, Wang $\mathrm{YZ}, \mathrm{Xu} \mathrm{YH}$. Anti-tumor effect of beta-elemene in glioblastoma cells depends on p38 MAPK activation. Cancer Lett. 2008;264(1): $127-34$.

78 Li QQ, Wang G, Zhang M, Cuff CF, Huang L, Reed E. Beta-elemene, a novel plant-derived antineoplastic agent, increases cisplatin chemosensitivity of lung tumor cells by triggering apoptosis. Oncol Rep. 2009;22(1):161-70.

79 Li QQ, Lee RX, Liang H, Zhong Y. Anticancer activity of $\beta$-elemene and its synthetic analogs in human malignant brain tumor cells. Anticancer Res. 2013;33(1):65-76.

80 Liu JS, He SC, Zhang ZL, Chen R, Fan L, Qiu GL, et al. Anticancer effects of $\beta$-elemene in gastric cancer cells and its potential underlying proteins: a proteomic study. Oncol Rep. 2014;32(6):2635-47.

81 Silva MP, de Oliveira RN, Mengarda AC, Roquini DB, Allegretti SM, Salvadori MC, et al. Antiparasitic activity of nerolidol in a mouse model of schistosomiasis. Int J Antimicrob Agents. 2017;50(3):467-72.

82 Alonso L, Fernandes KS, Mendanha SA, Gonçalves PJ, Gomes RS, Dorta ML, et al. In vitro antileishmanial and cytotoxic activities of nerolidol are associated with changes in plasma membrane dynamics. Biochim Biophys Acta Biomembr. 2019;1861(6):1049-56.

83 Leite MC, Bezerra AP, de Sousa JP, Guerra FQ, Lima EO. Evaluation of antifungal activity and mechanism of action of citral against Candida albicans. Evid Based Complement Alternat Med. 2014;2014:378280.

84 Thomas ML, de Antueno R, Coyle KM, Sultan M, Cruickshank BM, Giacomantonio MA, et al. Citral reduces breast tumor growth by inhibiting the cancer stem cell marker ALDH1A3. Mol Oncol. 2016;10(9):1485-96. 
85 Shi C, Song K, Zhang X, Sun Y, Sui Y, Chen $\mathrm{Y}$, et al. Antimicrobial activity and possible mechanism of action of citral against Cronobacter sakazakii. PLoS One. 2016;11(7): e0159006.

86 Sheikh BY, Sarker MMR, Kamarudin MNA, Mohan G. Antiproliferative and apoptosis inducing effects of citral via p53 and ROS-induced mitochondrial-mediated apoptosis in human colorectal HCT116 and HT29 cell lines. Biomed Pharmacother. 2017;96:834-46.

87 Sanches LJ, Marinello PC, Panis C, Fagundes TR, Morgado-Díaz JA, de-Freitas-Junior JC, et al. Cytotoxicity of citral against melanoma cells: the involvement of oxidative stress generation and cell growth protein reduction. Tumour Biol. 2017;39(3):1010428317695914.

88 Zielińska A, Martins-Gomes C, Ferreira NR, Silva AM, Nowak I, Souto EB. Anti-inflammatory and anti-cancer activity of citral: optimization of citral-loaded solid lipid nanoparticles (SLN) using experimental factorial design and LUMiSizer. Int J Pharm. 2018;553(1-2):428-40.

89 Kremer JL, Melo GP, Marinello PC, Bordini HP, Rossaneis AC, Sábio LR, et al. Citral prevents UVB-induced skin carcinogenesis in hairless mice. J Photochem Photobiol B Biol. 2019;198:111565.

90 Nigjeh SE, Yeap SK, Nordin N, Rahman H, Rosli R. In vivo anti-tumor effects of citral on $4 \mathrm{~T} 1$ breast cancer cells via induction of apoptosis and downregulation of aldehyde dehydrogenase activity. Molecules. 2019;24(18): 3241.

91 Tisserand R, Young R. Essential oil safety: a guide for health care professionals. 2nd ed. London: Elsevier; 2014. p. 780.

92 Karlberg AT, Magnusson K, Nilsson U. Air oxidation of d-limonene (the citrus solvent) creates potent allergens. Contact Derm. 1992; 26(5):332-40.

93 Bråred Christensson J, Karlberg AT, Andersen KE, Bruze M, Johansen JD, Garcia-Bravo $\mathrm{B}$, et al. Oxidized limonene and oxidized linalool: concomitant contact allergy to common fragrance terpenes. Contact Derm. 2016; 74(5):273-80.

94 Russo EB. Taming THC: potential cannabis synergy and phytocannabinoid-terpenoid entourage effects. Br J Pharmacol. 2011;163(7): 1344-64.

95 Kovalchuk I, Pellino M, Rigault PO, van Velzen R, Ebersbach J, Ashnest JR, et al. The genomics of cannabis and its close relatives. Ann Rev Plant Biol. 2020;71:713-739.

96 Allen KD, McKernan K, Pauli C, Roe J, Torres A, Gaudino R. Genomic characterization of the complete terpene synthase gene family from Cannabis sativa. PLos One. 2019;14(9): e0222363.

97 Booth JK, Page JE, Bohlmann J. Terpene synthases from Cannabis sativa. PLos One. 2017; 12(3):e0173911.

98 Gunnewich N, Page JE, Kollner TG, Degenhardt J, Kutchan TM. Functional expression and characterization of trichome-specific $(-)$-limonene synthase and (+)- $\alpha$-pinene synthase from Cannabis sativa. Nat Prod Commun. 2007;2(3):223-32.

99 Rice S, Koziel JA. Characterizing the smell of marijuana by odor impact of volatile compounds: an application of simultaneous chemical and sensory analysis. PLos One. 2015;10(12):e0144160.

100 Hazekamp A, Tejkalová K, Papadimitriou S. Cannabis: from cultivar to chemovar II-A metabolomics approach to cannabis classification. Cannabis Cannabinoid Res. 2016; 1(1):202-15.

101 Gallily R, Yekhtin Z, Hanuš LO. The antiinflammatory properties of terpenoids from cannabis. Cannabis Cannabinoid Res. 2018; 3(1):282-90.

102 Downer EJ. Anti-inflammatory potential of terpenes present in Cannabis sativa L. ACS Chem Neurosci. 2020;11(5):659-62.

103 Baron EP. Medicinal properties of cannabinoids, terpenes, and flavonoids in cannabis, and benefits in migraine, headache, and pain: an update on current evidence and cannabis science. Headache. 2018;58(7): 1139-86.

104 Nuutinen T. Medicinal properties of terpenes found in Cannabis sativa and Humulus lupulus. Eur J Med Chem. 2018;157:198228.

105 Namdar D, Voet H, Ajjampura V, Nadarajan S, Mayzlish-Gati E, Mazuz M, et al. Terpenoids and phytocannabinoids co-produced in Cannabis sativa strains show specific interaction for cell cytotoxic activity. Molecules. 2019;24(17):3031.

106 Ferber SG, Namdar D, Hen-Shoval D, Eger G, Koltai H, Shoval G, et al. The "entourage effect": terpenes coupled with cannabinoids for the treatment of mood disorders and anxiety disorders. Curr Neuropharmacol. 2020;18(2):87-96.

107 Finlay DB, Sircombe KJ, Nimick M, Jones C, Glass M. Terpenoids from cannabis do not mediate an entourage effect by acting at cannabinoid receptors. Front Pharmacol. 2020; 11:359.

108 Venditti A, Frezza C, Sciubba F, Serafini M, Bianco A, Cianfaglione $\mathrm{K}$, et al. Volatile components, polar constituents and biological activity of tansy daisy (Tanacetum macrophyllum (Waldst. et Kit.) Schultz Bip.). Ind Crops Prod. 2018;118:225-35.

109 Kiso Y, Tohkin M, Hikino H. Antihepatotoxic principles of Atractylodes rhizomes. J Nat Prod. 1983 Sep-Oct;46(5):651-4.

110 Ma EL, Li YC, Tsuneki H, Xiao JF, Xia MY, Wang MW, et al . Beta-eudesmol suppresses tumour growth through inhibition of tumour neovascularisation and tumour cell proliferation. J Asian Nat Prod Res. 2008 Jan-Feb;10(1-2):159-67.

111 Tsuneki H, Ma EL, Kobayashi S, Sekizaki N, Maekawa K, Sasaoka T, et al. Antiangiogenic activity of beta-eudesmol in vitro and in vivo. Eur J Pharmacol. 2005 Apr 11;512(23):105-15.

112 Bomfim DS, Ferraz RP, Carvalho NC, Soares MB, Pinheiro ML, Costa EV, et al. Eudesmol isomers induce caspase-mediated apoptosis in human hepatocellular carcinoma HepG2 cells. Basic Clin Pharmacol Toxicol. 2013 Nov;113(5):300-6.

$113 \mathrm{Lu} \mathrm{Y-C.} \mathrm{Studies} \mathrm{on} \mathrm{the} \mathrm{chemical} \mathrm{constitu-}$ ents of the essential oil of Rhododendron tsinghaiense Ching. Huaxue Xuebao 1980; 38(3):241-9.

114 Cavalieri E, Mariotto S, Fabrizi C, de Prati AC, Gottardo R, Leone S, et al. Alpha-bisabolol, a nontoxic natural compound, strongly induces apoptosis in glioma cells. Biochem Biophys Res Commun. 2004 Mar 12;315(3): 589-94.

115 Darra E, Lenaz G, Cavalieri E, Fato R, Mariotto S, Bergamini C, et al. Alpha-bisabolol: unexpected plant-derived weapon in the struggle against tumour survival? Ital J Biochem. 2007 Dec;56(4):323-8.

116 Darra E, Abdel-Azeim S, Manara A, Shoji K Maréchal JD, Mariotto S, et al. Insight into the apoptosis-inducing action of alpha-bisabolol towards malignant tumor cells: involvement of lipid rafts and Bid. Arch Biochem Biophys. 2008 Aug 15;476(2):113-23.

117 Rigo A, Ferrarini I, Lorenzetto E, Darra E, Liparulo I, Bergamini C, et al. BID and the a-bisabolol-triggered cell death program: converging on mitochondria and lysosomes. Cell Death Dis. 2019 Nov 26;10(12):889.

118 Anter J, Romero-Jiménez M, FernándezBedmar Z, Villatoro-Pulido M, Analla M, Alonso-Moraga A, et al. Antigenotoxicity, cytotoxicity, and apoptosis induction by apigenin, bisabolol, and protocatechuic acid. J Med Food. 2011 Mar;14(3):276-83.

119 Fernandes ES, Passos GF, Medeiros R, da Cunha FM, Ferreira J, Campos MM, et al Anti-inflammatory effects of compounds alpha-humulene and (-)-trans-caryophyllene isolated from the essential oil of Cordia verbenacea. Eur J Pharmacol. 2007 Aug 27; 569(3):228-36.

120 Volcho KP, Laev SS, Ashraf GM, Aliev G, Salakhutdinov NF. Application of monoterpenoids and their derivatives for treatment of neurodegenerative disorders. Curr Med Chem. 2018;25(39):5327-46.

121 Buczynski MW, Parsons LH. Quantification of brain endocannabinoid levels: methods, interpretations and pitfalls. Br J Pharmacol. 2010;160(3):423-42.

122 Fischedick JT, Hazekamp A, Erkelens T, Choi YH, Verpoorte R. Metabolic fingerprinting of Cannabis sativa L., cannabinoids and terpenoids for chemotaxonomic and drug standardization purposes. Phytochemistry. 2010;71(17-18):2058-73

123 Casano S, Grassi G, Martini V, Michelozzi $M$. Variations in terpene profiles of different strains of cannabis sativa L. Acta Hortic 2011;925(925):115-21. 